\title{
Climate Change and Cereal Production Evolution Trend in the Sahel: Case Study in Mali from 1951 to 2010
}

\author{
M. Kouressy ${ }^{1}$, B. Sultan ${ }^{2}$, M. Vaksmann ${ }^{3,7}$, J. F. Belières ${ }^{4,8}$, L. Claessens ${ }^{5}$, M. Dingkuhn ${ }^{6,7} \&$ N. Témé ${ }^{1}$ \\ ${ }^{1}$ Institut d'Economie Rurale (IER), Laboratoire Sol Eau Plante, Mali \\ ${ }^{2}$ ESPACE-DEV, Univ Montpellier, IRD, Univ Guyane, Univ Reunion, Univ Antilles, Univ Avignon, Maison de \\ la Télédétection, 500 rue Jean-François Breton, F-34093 Montpellier Cedex, France \\ ${ }^{3}$ CIRAD, UMR AGAP, BP 1813, Bamako, Mali \\ ${ }^{4}$ CIRAD, UMR ART-DEV, F-34398, Ampandrianomby, BP 853, Antananarivo, Madagascar \\ ${ }^{5}$ International Institute of Tropical Agriculture (iita.org), Arusha, Tanzania \\ ${ }^{6}$ CIRAD, UMR AGAP, F-34398, Montpellier, France \\ ${ }^{7}$ AGAP, Univ. Montpellier, CIRAD, INRA, Montpellier SupAgro, Montpellier, France \\ ${ }^{8}$ UMR ART-DEV, Univ Montpellier, Montpellier, France \\ Correspondence: M. Kouressy, Institut d'Economie Rurale (IER), Laboratoire Sol Eau Plante, BP 262 Sotuba, \\ Bamako, Mali. E-mail: nanym63@gmail.com
}

\author{
Received: February 19, 2019 Accepted: March 15, 2019 Online Published: April 9, 2019 \\ doi:10.5539/sar.v8n2p68 \\ URL: https://doi.org/10.5539/sar.v8n2p68
}

\begin{abstract}
Mali is a Sahelian country with a large climatic contrast from North to South. The current climatic and production evolutionary study is focused on the six major agro-climatic cereal production zones ranging from Kayes $(400 \mathrm{~mm})$ to Sikasso $(>1000 \mathrm{~mm})$ of rainfalls. Climatic data are rainfall records, daily maximum and minimum temperatures from 60 years of the six major synoptic weather observation stations. Data were analyzed on comparing average decades of the two normal periods of 30 years (1951-1980) and (1981-2010). Annual agronomic production data for millet, sorghum, maize and rice are derived from Mali's agricultural statistics base from 1984 to 2013. Main climatic results analyses indicate that climate change resulted in a decrease of $100 \mathrm{~mm}$ isohyets between the 2 periods of 30 years. The structure of the rainy season was little changed between these two periods since the average start of the season was delayed by 6 days and the average end date of the season became earlier by 4 days. Maximum temperatures increased significantly from $+0.44^{\circ} \mathrm{C}$ to $+1.53^{\circ} \mathrm{C}$ and minimum temperatures significantly increased from $+1.05^{\circ} \mathrm{C}$ to $+1.93^{\circ} \mathrm{C}$ in varying way depending on the sites. Statistics of major agronomic food crop production in Mali from 1984 to 2013 indicate an average increase of 985 to 4492 thousand tones, or $22 \%$ increase per year. There is a positive upward in saw tooth trend in Malian production from 1984 to 2013. This positive trend is the result of a combination of agricultural extension, agronomic research application and the management of small farmer holder in the Sahel. This evolution needs better study for drawing necessary right conclusions.
\end{abstract}

Keywords: rainfalls, food crop, millet, sorghum, maize, rice, production, climate change, temperatures, Sahel

\section{Introduction}

Mali is one of the largest countries in West Africa. It is a landlocked and located between 10 and 25 degrees north latitude. Its population, which was 7,620,000 in 1987, increased to $17,467,108$ million in 2016. This population accounts for more than $80 \%$ of the agricultural sector. Considered as West Africa breadbasket, Mali is divided into four climatic zones along the north-south gradient:

- The Saharan climate in the north, occupies $2 / 3$ of the country, with rainfall ranging from 50 to $250 \mathrm{~mm}$ per year. Windy alluvial floodplains and sand dunes cover the landscape. Clay soils are more or less sodic and sterile (Bertrand \& Gigou, 2000).

- The Sahelian climate extends from the Atlantic coast of West Africa to Sudan and is limited by isohyets 100 $\mathrm{mm}$ in the north and $600 \mathrm{~mm}$ in south, of annual rainfalls (De Vries, F.W.T. \& Djiteye, 1982). In Mali, the 
Sahelian climate goes from East of Senegal border to West of Niger border.

- The Sudanese climate (south of the Sahelian zone) has an average of 700-900 mm yearly rainfall.

- The Sudano-Guinean climate has a yearly average rainfall ranging from 1000 to over $1200 \mathrm{~mm}$ in the south of the country (Bertrand \& Gigou, 2000).

Areas, such as the Sahel zones, were hit by series of rainfall deficits in 1968, 1972, 1983 and 1984. These years of black episodes for the Sahelian population had been justified by the scientific community as a crisis related to the Sahelian climate known by its aridity, the destruction of poor soils under the effect of excessive grazing linked to human activity (Boudet, 1972). It has been called "drought in the Sahel". However the drying process does not date from our era but is around 2500 BC. J.C. (Klaus \& S., 1992).

Nowadays, climate change phenomenon is global with alarming episodes of other climate parameters such as temperature, we talk of climate change. Global warming was first discussed and modeled for the first time (Arrhenius, 1896). Since then, several researchers have focused on climate change to identify scientific, technical and socio-economic information needed to understand climate change (De Vries et al., 1982).

Since agriculture birth, man has always been interested in his bioclimatic environment. The complexity of the climate in its diversity and in relation to the various factors influencing its evolution and its variation make that the climatic phenomenon exceeds men memory capacities or the popular conscience which records only the events of natural disasters (great famine, great flood, heat waves, hurricanes etc.). The literature reports series of important droughts $(1680,1750,1820,1830,1910,1914,1930,1940,1944$ and with its worse burden and duration, the great drought of 1968 to 1983). In order to overcome difficulties in memorizing climatic phenomena and to build up archival data for statistical purposes, recording of reliable climate data in space and time necessitated the creation of synoptic weather stations across worldwide including Mali. In this current paper, climate trends are studied using archived climatic data. These results will be cross-referenced with Mali agronomic data derived from its agricultural statistics to draw conclusions for the likely evolution of agricultural production in Mali.

\section{Materials and Method}

This paper examines the ten-year evolution of temperature, rainfall, seasonal structure in space and time over a period of 60 years broken down into two normal series of 30 years (1951-1980) and, 1981-2010). Climatic data analyzes are coupled with rain fed and irrigated crops production data such as millet (Pennisetum glaucum $(L$.) $R$. $B r$.), sorghum (Sorghum bicolor L. Moench), rice (Oriza sativa) and maize (Zea mays L.) in six contrasting agro ecologies along following north-south gradient in Mali.

\subsection{Meteorological Data}

Consist of maximum temperatures (TX), minimum temperatures (TN) and daily rainfall of six synoptic weather stations in Mali over the last 60 years. Comparable observation periods are two series of 30 years or normal year in agro climatology and come from the Mali National Agency of Meteorology (ANM). The first series of 1951-1980 corresponds to the onset of rainfall subsidence in the Sahel (drought of 1972-1973) and the second is the current phase impacted by climate change.

\section{Seasonal structure analysis (beginning and end of the rainy season) in Mali}

The determination of the start and end dates of rainy season were carried out from six synoptic stations according to the methodology described (Traoré S. B., Reyniers F. N., Vaksmann M., Koné B., Sidibé et al., 2000) (Traoré S. B. et al., 2000). These authors defined rainy season start time as the moment when, from May 1st, the water reserve reaches $30 \mathrm{~mm}$ without falling back below $15 \mathrm{~mm}$ within next 20 days. The end of the seasons corresponds to the moment when plants water requirement (ETR / ETP ratio between actual and potential evapotranspiration) definitely drops below $90 \%$. This is the time when rainfall does not compensate evapotranspiration. The end of the season usually corresponds to crop maturity phase which is completed thanks to water stored in the soil and with the last rains.

\section{Maximum and minimum temperatures}

Maximum and minimum temperatures were organized on ten year average basis following the two series of normal agro-climatic conditions. The first normal was from 1951-1980 and the second normal was 1981-2010. Pair wise statistical analysis comparison of each first normal decade of 1951-1980 to that of its second counterpart 1981-2010 (Table 1-12) was performed.

Six synoptic stations were studied: Nioro du Sahel $\left(15.14^{\circ} \mathrm{N}\right.$ latitude, $9.36^{\circ} \mathrm{W}$ longitude, altitude $\left.235 \mathrm{~m}\right)$, located in 400-500 mm rainfall zone; Ségou ( $13.24^{\circ} \mathrm{N}$ latitude, $6.09^{\circ} \mathrm{W}$ longitude, altitude $288 \mathrm{~m}$ ), located in 
isohyet 500-600 mm; Sotuba $\left(12.32^{\circ} \mathrm{N}\right.$ latitude, $7.57^{\circ} \mathrm{W}$ longitude, altitude $\left.320 \mathrm{~m}\right)$, located near the capital Bamako in isohyet $800-900 \mathrm{~mm}$; Koutiala $\left(12.24^{\circ} \mathrm{N}\right.$ latitude, $5.28^{\circ} \mathrm{W}$ longitude, altitude $\left.334 \mathrm{~m}\right)$, the former cotton basin in Mali located in isohyet $800-900 \mathrm{~mm}$; Sikasso $\left(11.21^{\circ} \mathrm{N}\right.$ latitude, $5.14^{\circ} \mathrm{W}$ longitude, altitude 271 $\mathrm{m}$ ) located in isohyet $900-1000 \mathrm{~mm}$; Bougouni $\left(12.25^{\circ} \mathrm{N}\right.$ latitude, $7.3^{\circ} \mathrm{C} \mathrm{O}$ longitude, altitude $\left.350 \mathrm{~m}\right)$ located in isohyet 1000-1200 mm Table 1.

\subsection{Agronomic Data}

Food crops production data are from the "Cellule de Planification et de la Statistique (CPS) " from the malian ministry of agriculture. Linear regression of cereal production against time was established on the average annual production of the 6 sites studied from 1984 to 2013.

Climate data were performed for each 30 years period on averages monthly ten days using XLS, Statgraphics and SigmaPlot software.

\section{Results Discussion}

\subsection{Potential Evolution Analysis of the Wet Season}

Table 1 gives sites and the two periods (1951-1980 and 1981-2010) trends for the beginning, the end of the season and total average rainfalls.

-For Nioro du Sahel synoptic station, the average total rainfall went from 573 to $459 \mathrm{~mm}$, corresponding to a 114 $\mathrm{mm}$ reduction in 30 years; which is statistically significant at the $5 \%$ level (Table 1). The average rainy season duration went from 63 to 53 days during the same period, resulting in 10 days of rain reduction per season in the rainy season potential; which is statistically significant at the $5 \%$ level.

- For Ségou synoptic station (Table 1), the average rainfall went from 710.2 to $614.7 \mathrm{~mm}$ while the length of the season decreased from 94 to 89 days; which is not statistically significant with 5 days reduction.

- For Sotuba (Bamako) synoptic station, the average rainfall decreased from $1010 \mathrm{~mm}$ to $883 \mathrm{~mm}$, a loss of 127 $\mathrm{mm}$ of rainfall. The decrease in total rainfall was statistically significant, whereas the rainy season duration was not and decreased from 127 to 124 days (Table 1) or 3 days reduction.

- For Koutiala synoptic station, the average rainfall was reduced from 994 to $853 \mathrm{~mm}$ which is statistically significant. The duration of the season also decreased from 137 to 121 days and is statistically significant (Table 1) with 16 days reduction.

- For Sikasso synoptic station, the average rainfall was reduced from 1239 to $1090 \mathrm{~mm}$, which is statistically significant. The duration of the season also decreased from 160 to 147 days and is statistically significant (Table 1) with 13 days reduction.

- For Bougouni synoptic station, the average rainfall was reduced from 1206 to $1111 \mathrm{~mm}$ which is statistically significant. The duration of the season also decreased from 161 to 150 days and is statistically significant (Table 1) with 11 days reduction.

Table 1. Declining rainfall and seasonal structure periods (1951-1980) and (1981-2010) in the 6 sites in Mali

\begin{tabular}{|c|c|c|c|c|c|c|c|c|c|c|c|c|c|c|}
\hline Buffer (mm) & Sites & Latitude & Longitude & Altitude $(\mathrm{m})$ & Periods & Onset season & SD & End of season & SD & Duration (days) & Means rainfall $(\mathrm{mm})$ & SD & H.G & S. Level \\
\hline \multirow[b]{2}{*}{$400-500$} & \multirow[b]{2}{*}{ Nioro du Sahel } & \multirow[b]{2}{*}{$15.14 ; \mathrm{N}$} & \multirow[b]{2}{*}{$9.36 ; 0$} & \multirow[b]{2}{*}{235} & 1951-1980 & $15 / 7$ & 13.8 & $15 / 9$ & 10.5 & 63 & 572.7 & 157.0 & A & \multirow[b]{2}{*}{$s$} \\
\hline & & & & & $1981-2010$ & $21 / 7$ & 18.1 & $12 / 9$ & 11.1 & 53 & 459.1 & 107.0 & B & \\
\hline & & & & & & & & \multicolumn{2}{|c|}{ Difference in } & 10 & 113.6 & & & \\
\hline \multirow[b]{2}{*}{$600-700$} & \multirow[b]{2}{*}{ Segou } & \multirow[b]{2}{*}{$13.24 ; \mathrm{N}$} & \multirow[b]{2}{*}{$6.09 ; 0$} & \multirow[b]{2}{*}{288} & $1951-1980$ & $23 / 6$ & 17.1 & $25 / 9$ & 12.6 & 94 & 710.2 & 116.0 & A & \multirow[b]{2}{*}{ NS } \\
\hline & & & & & $1981-2010$ & $24 / 6$ & 20.2 & $21 / 9$ & 9.4 & 89 & 614.7 & 140.0 & A & \\
\hline & & & & & & & & \multicolumn{2}{|c|}{ Difference in } & 5 & 95.5 & & & \\
\hline \multirow[b]{2}{*}{$800-900$} & \multirow[b]{2}{*}{ Sotuba } & \multirow[b]{2}{*}{$12.32 ; \mathrm{N}$} & \multirow[b]{2}{*}{$7.57 ; 0$} & \multirow[b]{2}{*}{320} & $1951-1980$ & $5 / 6$ & 13.8 & $10 / 10$ & 10.4 & 127 & 1010.0 & 188.0 & A & \multirow[b]{2}{*}{$\mathrm{s}$} \\
\hline & & & & & $1981-2010$ & $6 / 6$ & 18.8 & $8 / 10$ & 11.2 & 124 & 883.0 & 155.0 & B & \\
\hline & & & & & & & & \multicolumn{2}{|c|}{ Difference in } & 3 & 127.0 & & & \\
\hline \multirow[b]{2}{*}{$800-900$} & \multirow[b]{2}{*}{ Koutiala } & \multirow[b]{2}{*}{$12.24 ; \mathrm{N}$} & \multirow[b]{2}{*}{$5.28 ; 0$} & \multirow[b]{2}{*}{334} & 1951-1980 & $26 / 5$ & 16.0 & $10 / 10$ & 11.5 & 137 & 994.0 & 190.0 & A & \multirow[b]{2}{*}{ s } \\
\hline & & & & & $1981-2010$ & $5 / 6$ & 14.8 & $4 / 10$ & 11.7 & 121 & 853.0 & 178.0 & B & \\
\hline & & & & & & & & \multicolumn{2}{|c|}{ Difference in } & 16 & 141.0 & & & \\
\hline \multirow[b]{2}{*}{$1000-1200$} & \multirow[b]{2}{*}{ Sikasso } & \multirow[b]{2}{*}{$11.21 ; \mathrm{N}$} & \multirow[b]{2}{*}{$5.41 ; 0$} & & $1951-1980$ & $9 / 5$ & 20.2 & $16 / 10$ & 14.4 & 160 & 1239.0 & 183.0 & A & \\
\hline & & & & 271 & $1981-2010$ & $20 / 5$ & 16.0 & $14 / 10$ & 9.2 & 147 & 1090.0 & 170.0 & B & $\mathrm{s}$ \\
\hline & & & & & & & & Diff & rence in & 13 & 149.0 & & & \\
\hline & & & & & 1951-1980 & $10 / 5$ & 19.8 & $18 / 10$ & 13.1 & 161 & 1206.0 & 203.0 & $\mathbf{A}$ & \\
\hline $1000-1200$ & Bougouni & $11.25 ; \mathrm{N}$ & $7.3 ; 0$ & 350 & $1981-2010$ & $15 / 5$ & 17.6 & $12 / 10$ & 8.9 & 150 & 1111.0 & 196.0 & A & NS \\
\hline & & & & & & & & Diff & rence in & 11 & 95.0 & & & \\
\hline
\end{tabular}

Observations: develop abbreviations: $\mathrm{SD}=$ standard deviation, $\mathrm{HG}=$ homogenous group $\mathrm{S}$. level = significance level $(P=0.05$. $)$ Rain season onset. End of rainy season. 


\subsection{Sites Maximum and Minimum Temperatures Evolution Analysis}

Over the two periods, TX, TN have evolved as follows:

- In Nioro du Sahel, the maximum temperature evolved from 1951 to 1980 to $40.03^{\circ} \mathrm{C}$ and from 1981 to 2010 to $40.42^{\circ} \mathrm{C}$ with a difference of $0.53^{\circ} \mathrm{C}$ between the two normal on average over all decades. Differences are statistically significant (Figure 1-a, Table 2; 3). The minimum temperature for the same periods evolved from 20.71 to $21.53^{\circ} \mathrm{C}$ with a statistical difference of $0.82^{\circ} \mathrm{C}$ between the two normal.

- In Ségou, for the same period, the maximum temperature evolved from 34.59 to $36.16^{\circ} \mathrm{C}$, a difference of $1.57^{\circ} \mathrm{C}$ which is statistically significant. The minimum temperature ranged from 21.21 to $22.7^{\circ} \mathrm{C}$ with a difference of $1.49^{\circ} \mathrm{C}$. This is highly significant (Figure 1-b, Table $4 ; 5$ ).

-For Sotuba (Bamako), the maximum temperature varied from 34.41 to $34.85^{\circ} \mathrm{C}$, an increase of $0.44^{\circ} \mathrm{C}$ which is statistically significant. The minimum ranged from 19.82 to $20.87^{\circ} \mathrm{C}$, an increase of $1.05^{\circ} \mathrm{C}$ which is highly significant (Figure 1-c, Table 6; 7).

- In Koutiala, the maximum varied from 34.11 to $34.3^{\circ} \mathrm{C}$, a difference of $0.19^{\circ} \mathrm{C}$ which is not statistically significant. The minimum ranged from 19.75 to $21.68^{\circ} \mathrm{C}$, a difference of $1.93^{\circ} \mathrm{C}$, which is highly significant (Figure 2-d, Table 8; 9) and the highest of all sites.

- Sikasso shows a variation of its maximum temperature from 33.62 to $33.9^{\circ} \mathrm{C}$, a difference of $0.28^{\circ} \mathrm{C}$ which is significant. Minimum temperature varied from 20.12 to $21.37^{\circ} \mathrm{C}$, an increase of $1.25^{\circ} \mathrm{C}$ which is highly significant (Figure 2- e, Table 10; 11).

- At Bougouni, the maximum temperature ranged from 33.51 to $34.36^{\circ} \mathrm{C}$, an increase of $0.85^{\circ} \mathrm{C}$, which is statistically significant. The minimum temperature varied from 20.53 to $21.21^{\circ} \mathrm{C}$, an increase of $0.68^{\circ} \mathrm{C}$, which is statistically significant (Figure 2-f, Table 12; 13). 
a ) Nioro du Sahel Temperature

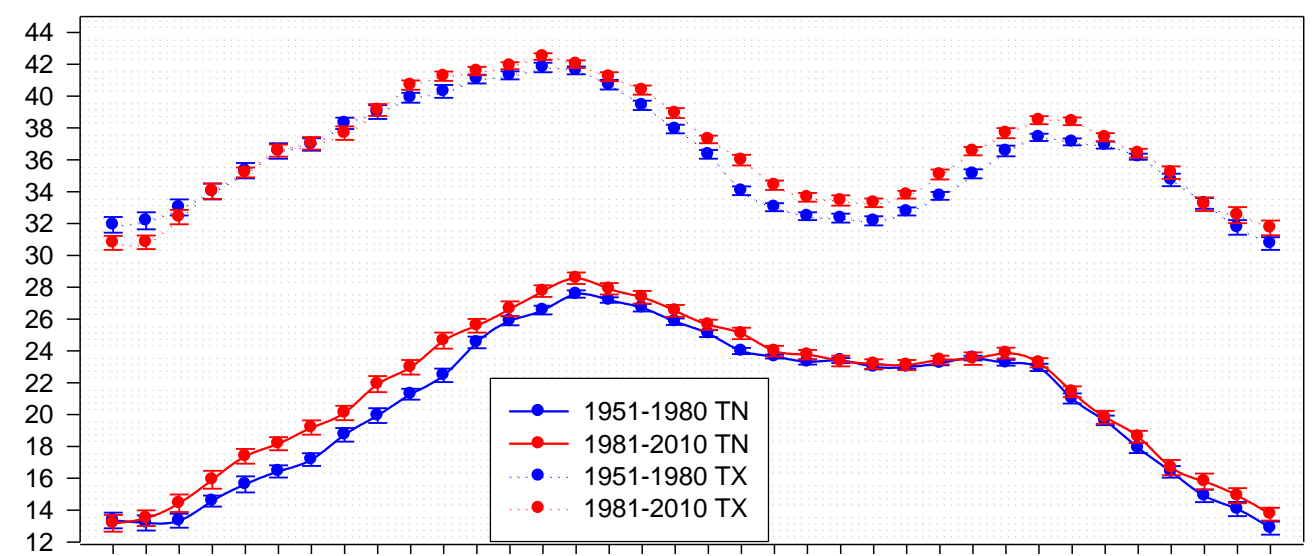

b) Segou Temperature

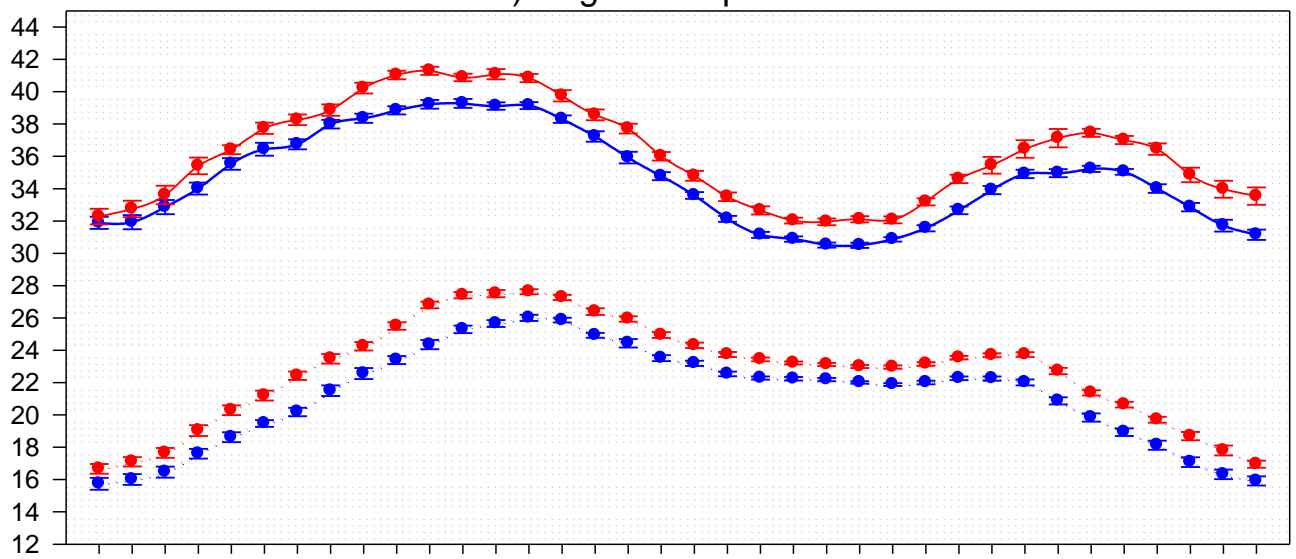

c) Sotuba Temperature

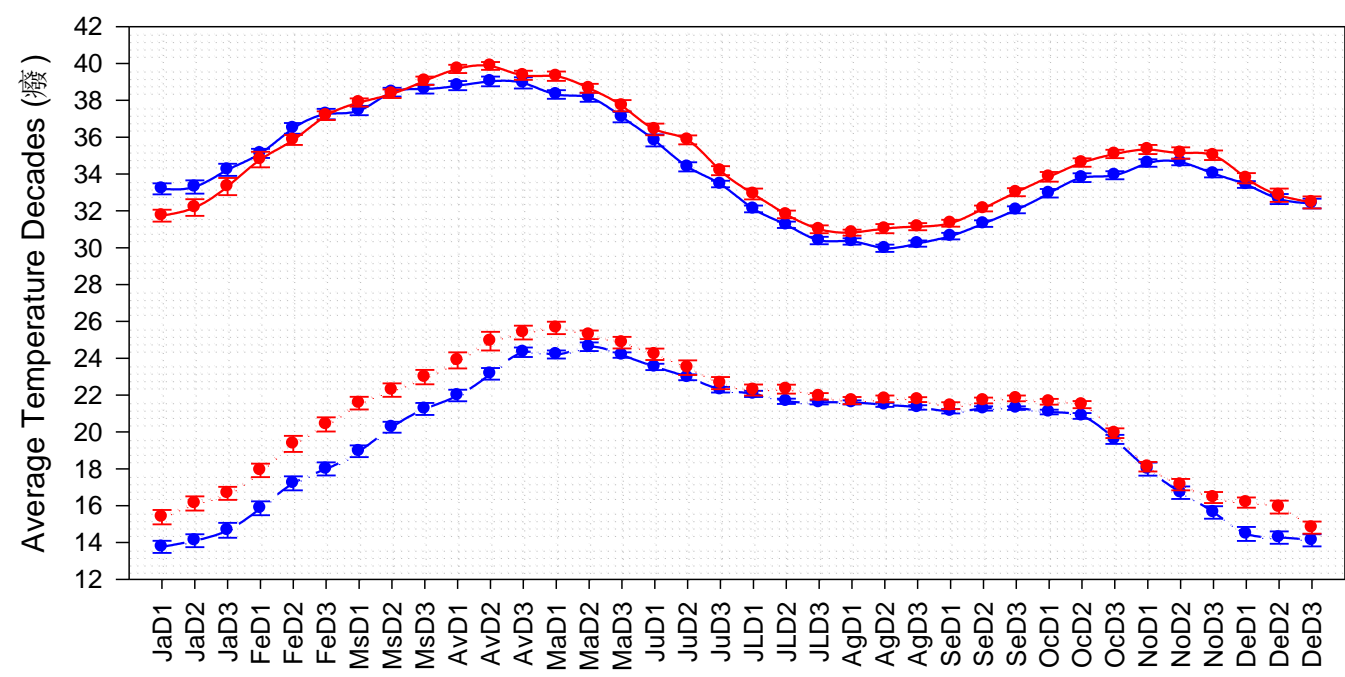

Decades month

Figure 1. a-b-c: averages maximum (TX) and minimum (TN) temperatures of 1951-1980 normal compared to those of 1981-2010 normal at Nioro, Ségou and Sotuba (Bamako) meteorological synoptic stations in Mali 


\section{d) Koutiala Temperature}

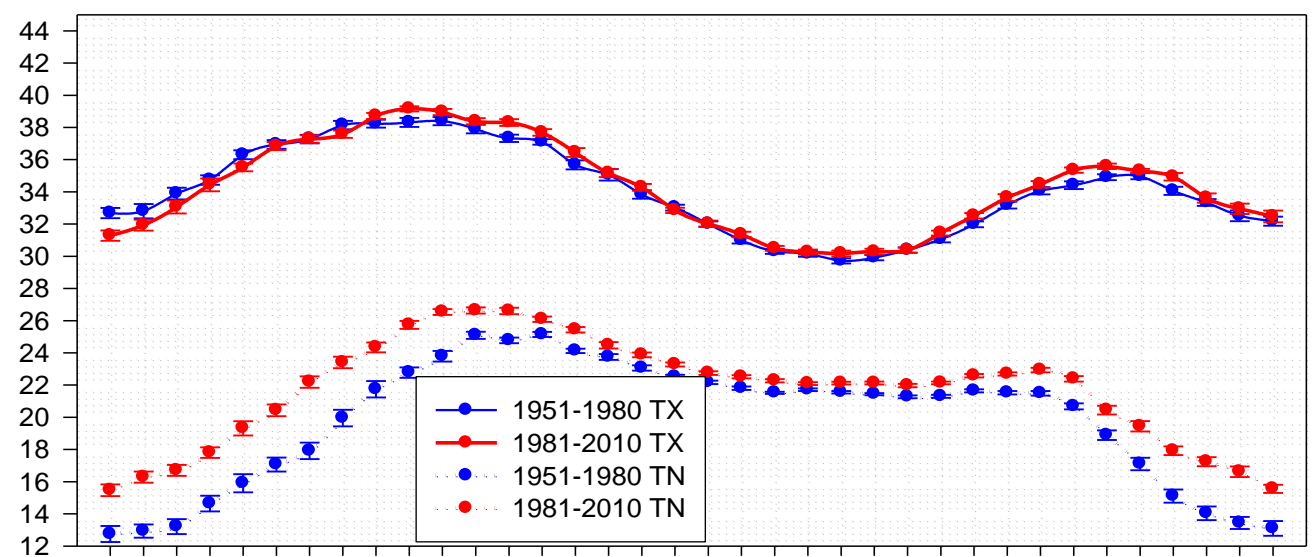

e ) Sikasso Temperature

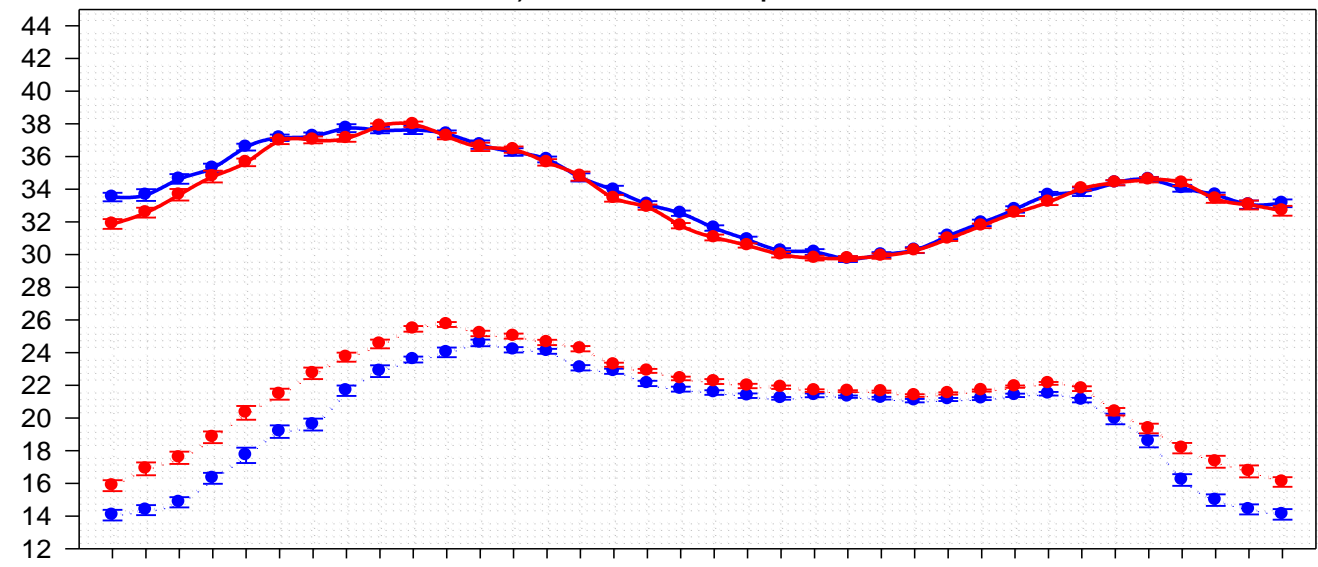

f) Bougouni Temperature

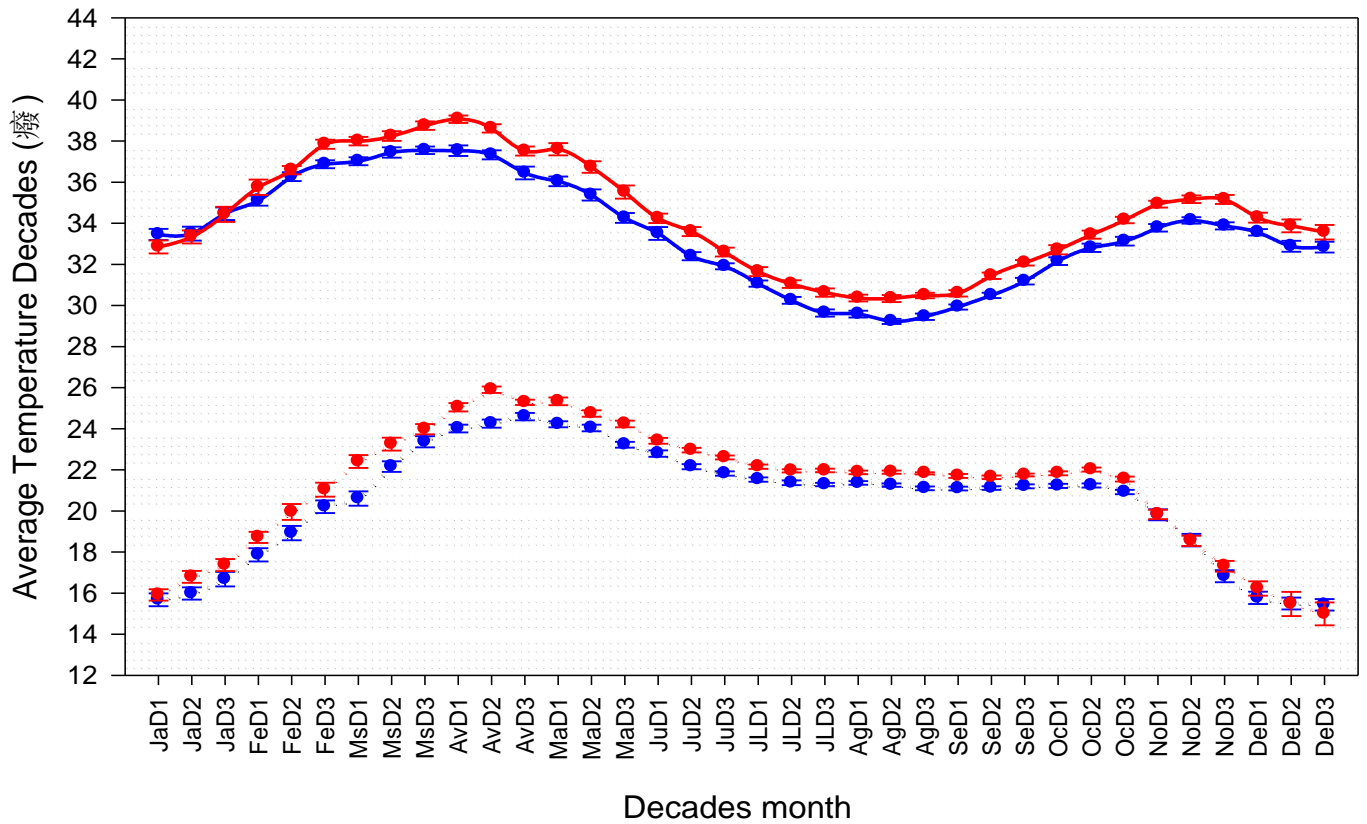

Figure 2. d-e-f: averages maximum (TX) and minimum (TN) temperatures of 1951-1980 normal compared to those of 1981-2010 normal at Koutiala, Sikasso and Bougouni 


\section{Discussion and Interpretation}

Seasonal structure data from studied sites in Mali (Table 1) indicate an average delay in rainy season onset date with varying extent from one site to another: an average delay of 7 days in Nioro du Sahel, 9 days in Koutiala and Sikasso and 5 days in Bougouni. The end of the season is less variable. These results are consistent with those of (Kouressy, Dingkuhn, Vaksmann \& Heinemann, 2008) and correspond to an average retreat of isohyets of $100 \mathrm{~mm}$ per site. It is noted that the seasonal structure of Ségou region was statistically less disturbed compared to those of the other sites during the last 30 years. In general, there is a widespread drying tendency over time and space along the north-south latitudinal gradient in Mali. Rainfalls decreased from the 1970s (Bates, Z. W. K., S. Wu \& Palutikof, 2008) but this decrease did not affect rainy season length (De Rouw, 2004). Total rainfalls collapse and early and late rainy season variability (Table 1) confirm findings these authors works (Courel, 1984; S. Salack, B. Muller \& Gaye, 2011; Traoré, Kouressy, Vaksmann, Tabo, Maikano et al., 2007; Traoré S. B. et al., 2000)

Regarding average temperature evolutions, there is a significant increase in maximum temperatures between the two normal periods and specially a strong increase in minimum temperatures. This trend is consistent with the climate results obtained in Senegal (Maurizio, D. \& Massimiliano, 2013). These results support popular beliefs in Mali that differences in temperatures between seasons are less marked now and say they are currently in a continuous heat throughout the year contrary to the past where temperature changes in the Sahel were well marked between cold and warm seasons. Current analyses are consistent with scientific information on warming in the Sahel (Arona, Adeline, Richard, SoniaI, David et al., 2018; Wallace, 1975) and in some areas of Mali (Traoré, Marc, Mark, Mariana \& Ken, 2013). It is noted that the temperature curve is bimodal opposed to the uni-modal rainfall curve, in Mali. This can be easily explained by the rainfall effect, which decreases temperature during the cropping season (Gigou, Giraudy, Doucouré, Healy, Traoré et al., 2004).

Our agronomic results (Fig. 8) invalidate production predictions decrease in the Sahel due to decreased rainfall (Bates et al., 2008).

Agronomic data (Figure 7-a-b-c-d, and 8) from 1984 to 2013 indicate an increase in the average production of major cereals in the 6 sites at Mali from 985 to 4492 thousand tons, an increase of $22 \%$. This trend is confirmed by other researchers on other cereals (Jacques, Jean, Alain \& Gilles, 2013) and supports the conclusions (Martin K., Van L., Lenny G. J., van B. \& Joost Wolfa, 2016) on the ability of some African countries, including Mali, to ensure food self-sufficiency at horizon 2050.

Cereal production growth has therefore not been affected either by rising temperatures or decreasing rainfall. Several explanations for cereal production increase can be put forward:

-For Sahelian farmers, the variability of the beginning and end of the rainy season is greater than the actual amount of rainfall (Klaus \& S., 1992). Sahelian farmers have developed for adapting techniques to mitigate rainfall variability and drought effects (early planting date, diversity of cropping and cropping systems) to survive more than 200,000 years ago (Klaus \& S., 1992; Sissoko, Doumbia, Vaksmann, Hocdé, Bazile et al., 2008).

-The second aspect is species genetic diversity exploitation millet (Pennisetum glaucum (L.) R. Br.), sorghum (Sorghum bicolor L. Moench), rice (Oriza sativa) and maize (Zea mays L.) in small farm holders which seems to adapt to agro ecological conditions of the environment. Diversity of varieties species cropping in the same farming systems specific to each ecology, come from a "creative vision" of farmers responding to their socio-economic needs whatever the diversity of ecological production areas. Within each species, to improve production and minimize seasonal environmental variability effects. Sahelian farmers play on the morpho-botanical and physiological species diversities such as earliness and photoperiodism (Sultan, Guan, Kouressy, Biasutti, Piani et al., 2014; Sultan, Roudier, Quirion, Alhassane, Muller et al., 2013). In the Sahel, a farmers' experience is a case study for research to improve it and exploit in favor of smart and sustainable agriculture. These crops different agronomic traits, such as photoperiodism, enable crops to regulate their growth phase and synchronize their flowering at the end of the growing season to avoid biotic and abiotic pressures (mold, downpour pollen washing, bird damages) and promotes their maturity before end of season soil water depletion (Kouressy, 2002; Kouressy, Bazile, Vaksmann, Soumaré, Doucouré et al., 2003; Kouressy et al., 2008; Kouressy, Vaksmann, Niangado \& Sanogo, 2004; Kouressy, Vaksmann \& Sidibé, 2006; Vaksmann, Chantereau, Bahmani, Ag Hamada, Chartier et al., 1998; Vaksmann, Traoré \& Niangado, 1996). Other crops such as maize and rice are free from these biotic problems because of their floral biology (grains under husks and hull) so that their earliness is not an obstacle to their production, hence their acceptance by the Sahelian farmers. Specifically rice production has increased rapidly after the 2007-2008 food crises (Harold M. \& R., 2015). Obvious 
temperature rise results in Mali and the increase in production in the last 30 years let us conclude a delicate climate change compared to what would have been these adverse consequences on the agricultural production of small farmers in Mali (Figure 4).

\section{a ) Kayes area Crops production}

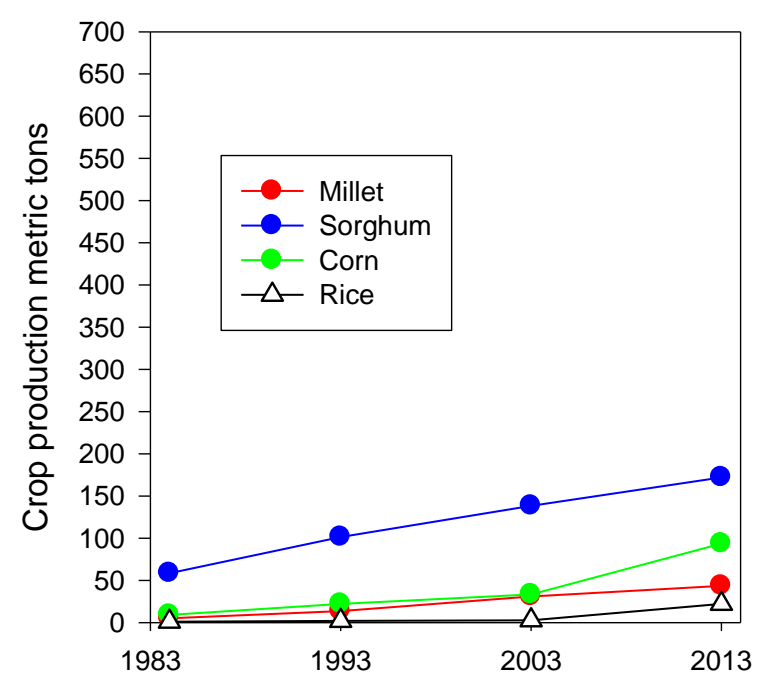

c ) Bamako area Crops production

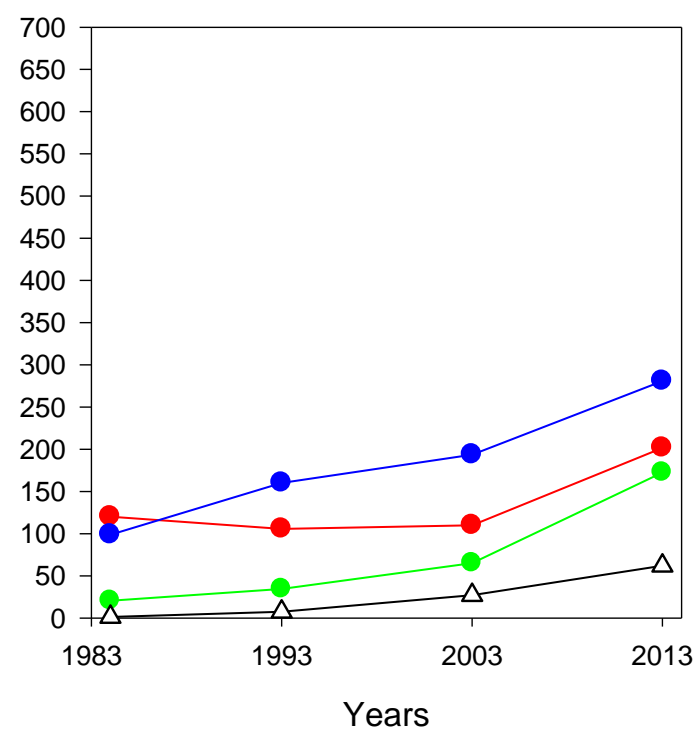

b ) Segou area Crops production

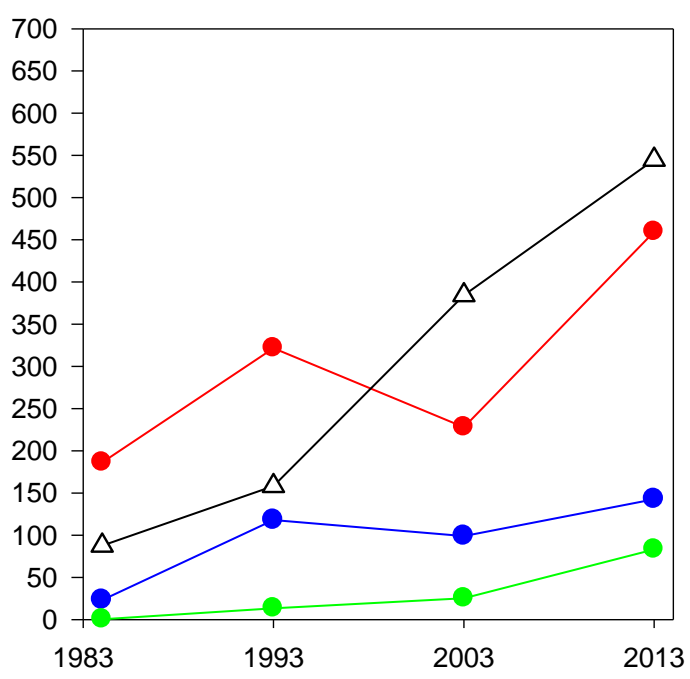

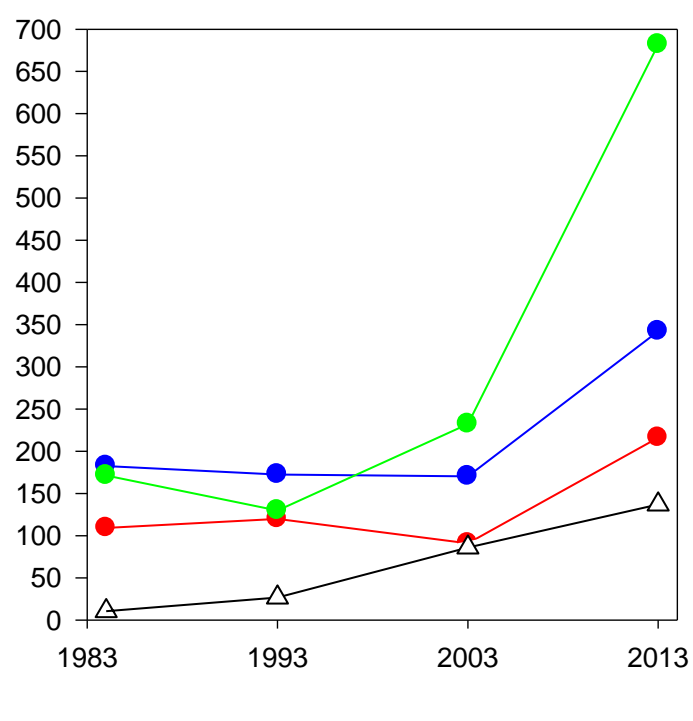

Figure 3. Evolution of millet, sorghum and maize production in thousand of tons of Kayes (a), Segou (b) Bamako (c) and Sikasso (d) regions from 1984 to 2013

Bamako is a district and is in Koulikoro region. 


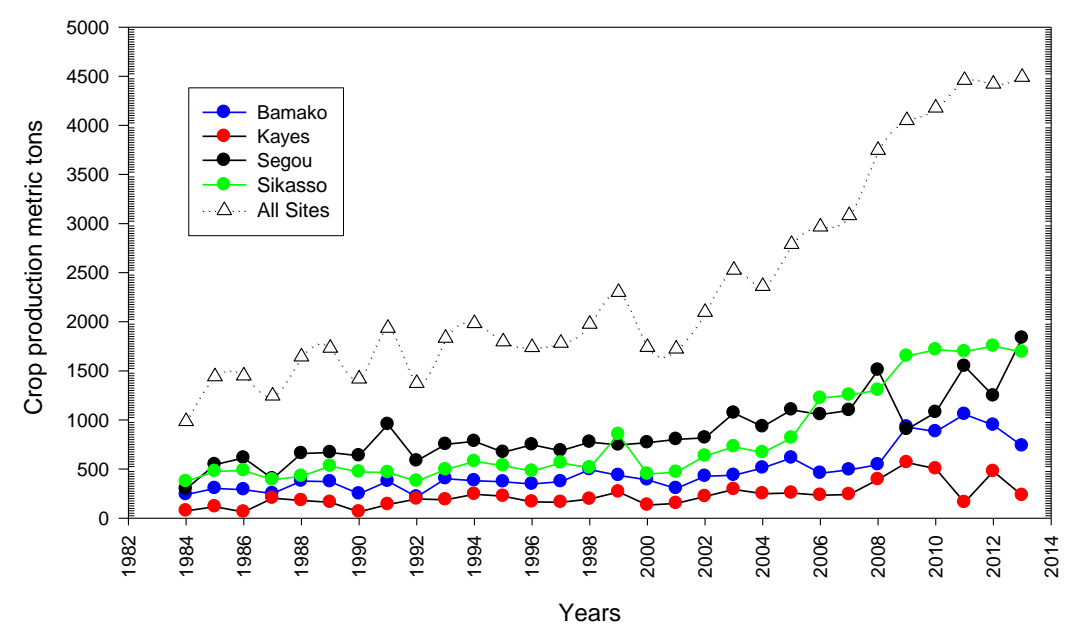

Figure 4. Evolution in rain fed total crops production in thousands of tons from 1984 to 2013 of sorghum, millet maize, rice of $4 \mathrm{r}$ regions (Kayes, Ségou, Bamako, and Sikasso) in Mali

\section{Conclusion}

Rainfall results analyses and temperature surveys indicate between the two periods (1951-1980 and 1981-2010), an average drawback of isohyets of between 95 and $150 \mathrm{~mm}$ for the studied sites (113 mm at Kayes, $95.5 \mathrm{~mm}$ in Ségou, $127 \mathrm{~mm}$ in Bamako, $137 \mathrm{~mm}$ in Koutiala, $149 \mathrm{~mm}$ in Sikasso, and $95 \mathrm{~mm}$ in Bougouni).

Maximum and minimum temperatures increased by $\left(+0.44{ }^{\circ} \mathrm{C},+1.05{ }^{\circ} \mathrm{C}\right) ;\left(+1.53{ }^{\circ} \mathrm{C},+1.4{ }^{\circ} \mathrm{C}\right) ;\left(+0.44{ }^{\circ} \mathrm{C},+\right.$ $\left.1.05{ }^{\circ} \mathrm{C}\right) ;\left(+0.19{ }^{\circ} \mathrm{C},+1.93{ }^{\circ} \mathrm{C}\right) ;\left(+0.28{ }^{\circ} \mathrm{C},+1.25^{\circ} \mathrm{C}\right) ;\left(+0.85^{\circ} \mathrm{C},+0.68{ }^{\circ} \mathrm{C}\right)$ respectively in Kayes, Ségou, Bamako, Koutiala, Sikasso and Bougouni.

There is a general trend towards drying out and increasing temperature in Mali. However, despite this situation, the agronomic results of cereal production are satisfactory. This leads us to hypothesize that our knowledge of understanding climate consequences are insufficient.

There is a positive saw tooth trend in production from 1984 to 2013 in Mali. This positive development is the result of a combination of the application of agricultural extension and agronomic research works and management at small farm holders in the Sahel. The average growth is $22 \%$. This evolution deserves better study for a better agricultural policy planning in Mali.

National production safety is essentially ensured by crop species choices according to their adaptation to the agro-climatic potentialities of the regions. This explains why in each production region, one species is dominant over the others that become complementary (Figure 3) and the sum of the production (Figure 8) of the whole constitutes a counter-random strategy of the climatic risk in Sahel guaranteeing food security.

\section{References}

Arona, D., Adeline, B., Richard, W., SoniaI, S., David, P. R., Mouhamadou, B. S., ... François, A. (2018). Changes in climate extremes over West and Central Africa at $1.5^{\circ} \mathrm{C}$ and $2{ }^{\circ} \mathrm{C}$ global warming. Environmental Research Letters, 13(6), 1-11.

Arrhenius, A. S. (1896). On the Influence of Carbonic Acid in the Air upon the Temperature of the Ground. Philosophical Magazine and Journal of Science Series 5, 41(251), 237-276. https://doi.org/10.1080/14786449608620846

Bates, B. C., Wu, S., \& Palutikof, J. P. (2008). Le changement climatique et l'eau. Secrétariat du GIEC. Genève, 236.

Bertrand, R., \& Gigou, J. (2000). La fertilité des sols tropicaux Livre. Ed. Maisonneuve et Larose.

Boudet, G. (1972). Desertification de l'Afrique tropicale sèche. ADANSONIA, ser. 2, 12(4), 4505-524.

Courel, M. F. (1984). Etude de l'évolution récente des milieux Sahélien à partir des mesures fournies par les satelites. Thèse de doctorat d'état Thesis, Paris I, 407 pp.

De Rouw, A. (2004). Improving yields and reducing risks in pearl millet farming in the African Sahel. 
Agricultural Systems, 81, 73-93. https://doi.org/10.1016/j.agsy.2003.09.002

De Vries, P. F. W. T., \& Djiteye, M. A. (1982). La productivité des pâturages saheliens. Une étude des sols, des végétations et de l'exploitation de cette ressource naturelle, Agricultural Research Report No. 918. Pudoc, Wageningen, Netherlands., pp. 525.

Gigou, J., Giraudy, F., Doucouré, C. O. T., Healy, S., Traoré, K. B., \& Guindo, O. (2004). L'âge des champs : un indicateur du passage de la culture itinérante à la culture permanente dans le bassin cotonnier du Mali. Cahiers Agricultures, 13, 467-472.

Harold, M., \& R., T. (2015). Les cultures céréalières: riz, maïs, millet, sorgho et blé. Nation Unies, Nourrir l'Afrique 21-23 octobre

Jacques, C., Jean, C., Alain, R., \& Gilles, T. (2013). Le sorgho. Éditions Quæ, CTA, Presses agronomiques de Gembloux1.245.

Klaus, M. L., \& S., K. (1992). Survivre au Sahel. Book(Un défi pour l'écologie et la politique de développement). $1-186$.

Kouressy, M. (2002). Etude de la durée du cycle des sorghos du Mali. Comparaison avec la durée de la saison des pluies. Evolution sur les 20 dernières années. DEA Thesis, Université du Mali, Bamako, 54 pp.

Kouressy, M., Bazile, D., Vaksmann, M., Soumaré, M., Doucouré, T., \& Sidibé, A. (2003). La dynamique des agroécosystèmes: un facteur explicatif de l'érosion variétale du sorgho - Le cas de la zone Mali-Sud. In: P. Dugué and P. Jouve (Editors), Organisation spatiale et gestion des ressources et des territoires ruraux (pp. 128-143). Actes du colloque international, Montpellier, France.

Kouressy, M., Dingkuhn, M., Vaksmann, M., \& Heinemann, A. B. (2008). Adaptation to diverse semi-arid environments of sorghum genotypes having different plant type and sensitivity to photoperiod. Agricultural and Forest Meteorology, 148, 357-371. https://doi.org/10.1016/j.agrformet.2007.09.009

Kouressy, M., Vaksmann, M., \& Sidibé, A. (2006). Diversité génétique, une stratégie de survie au Sahel, Actes du colloque de Troyes. La biodiversité végétale. Des plantes pour l'avenir., Troyes, France, pp. 353-354.

Kouressy, M., Vaksmann, M., Niangado, O., \& Sanogo, M., (2004). Valorisation et preservation de la diversité génétique du mil au Mali. In: G. Bezançon and J.L. Pham (Editors), Ressources génétiques des mils en Afrique de l'Ouest (pp. 45-58). IRD Editions, Niamey. https://doi.org/10.4000/books.irdeditions.539

Martin, K., Van, L., Lenny, G. J. van B., \& Joost, W. (2016). Can sub-Saharan Africa feed itself?. PNAS Early Edition, 1-6.

Maurizio, B., D., M., \& Massimiliano, P. (2013). CLIMAT Encadrement climatique et évaluation du changement climatique dans les régions d'étude Rapport, $\mathrm{N}^{\circ} 6$ Décembre1-142.

Salack, S., Muller, B., \& Gaye, A. T. (2011). Rain-based factors of high agricultural impacts over Senegal. Part I: integration of local to sub-regional trends and variability. Theor Appl Climatol 1061.22.

Sissoko, S., Doumbia, S., Vaksmann, M., Hocdé, H., Bazile, D., Sogoba, B., ... Dicko, B. G. (2008). Prise en compte des savoirs paysans en matière de choix variétal dans un programme de sélection. Cahier Agricultures, 17, 2128-132.

Sultan, B., Guan, K., Kouressy, M., Biasutti, M., Piani, C., Hammer, G. L., McLean, G. M., \& Lobell, D. B. (2014). Robust features of future climate change impacts on sorghum yields in West Africa. Environ. Res., Lett, 9(10), 1-13. https://doi.org/10.1088/1748-9326/9/10/104006

Sultan, B., Roudier, P., Quirion, P., Alhassane, A., Muller, B., Dingkuhn, M., Ciais, P., Guimberteau, M., Traore, P. S., \& Baron, C. (2013). Assessing climate change impacts on sorghum and millet yields in the Sudanian and Sahelian savannas of West Africa. Environ. Res., Lett., 8, 1-9. https://doi.org/10.1088/1748-9326/8/1/014040

Traoré, B., Marc, C., Mark, T. W., Mariana, C. R., \& Ken, E. G. (2013). Effects of climate variability and climate change on crop production in Southern Mali. European Journal of Agronomy, 49, 115-125. https://doi.org/10.1016/j.eja.2013.04.004

Traoré, P. C. S., Kouressy, M., Vaksmann, M., Tabo, R., Maikano, I., Traoré, S. B., \& Cooper, P. J. M. (2007). Climate prediction and agriculture: what is different about sudano-sahelian West Africa? In: M.V.K. Sivakumar and J. Hansen (Editors), Climate Prediction and Agriculture. Advances and Challenges. https://doi.org/10.1007/978-3-540-44650-7_19 
Traoré, S. B., Reyniers, F. N., Vaksmann, M., Koné, B., Sidibé, A., Yoroté, A., Yattara, K., \& M., K. (2000). Adaptation à la sécheresse des écotypes locaux de sorghos du Mali. Sécheresse, 11(4), 227-237.

Vaksmann, M., Chantereau, J., Bahmani, I., Ag Hamada, M., Chartier, M., \& Bonhomme, R. (1998). Influence of night temperature on photoperiod response of a west africain guinea sorghum landrace. In: C.J.é. Ratnadass A. (éd.), Gigou J. (éd.). (Editor), Amélioration du sorgho et de sa culture en Afrique de l'Ouest et du Centre. Atelier de Restitution du Programme Conjoint sur le Sorgho(pp. 23-28). CIRAD, Bamako, Mali.

Vaksmann, M., Traoré, S. B., \& Niangado, O. (1996). Le photopériodisme des sorghos africains. Agriculture et Développement, 913-18.

Wallace, B. S. (1975). Climatic Change: Are We on the Brink of a Pronounced Global Warming? Science, New Series, 189.

\section{Appendix A}

Table 2. Maximum temperature every ten days statistical analysis of the period 1951-1980 (30 years) and compared to the period 1981-2010 (30 years) at the meteorological station of Nioro du Sahel in Mali $\left(15.14^{\circ} \mathrm{N}\right.$ latitude, $9.36^{\circ} \mathrm{O}$ longitude; altitude $236 \mathrm{~m}$ )

\begin{tabular}{|c|c|c|c|c|c|c|c|c|}
\hline Periods & Month & Decades & Means & Diff. $\left({ }^{\circ} \mathrm{C}\right)$ & F-ratio & P-value & H. G. & S.Leve \\
\hline 1951-1980 & January & 1 & $\begin{array}{r}31.82 \\
\end{array}$ & & & & A & \\
\hline 1981-2010 & January & 1 & 30.78 & -1.04 & 2.85 & 0.0972 & $A$ & NS \\
\hline 1951-1980 & January & 2 & 32.17 & & & & A & \\
\hline $1981-2010$ & January & 2 & 30.82 & -1.35 & 3.81 & 0.0560 & A & NS \\
\hline 1951-1980 & January & 3 & 33 & & & & A & \\
\hline $1981-2010$ & January & 3 & 32.4 & -0.6 & 0.77 & 0.3854 & A & NS \\
\hline 1951-1980 & February & 4 & 34 & & & & A & \\
\hline $1981-2010$ & February & 4 & 34 & 0 & 0.00 & 0.9813 & A & NS \\
\hline $1951-1980$ & February & 5 & 35.32 & & & & A & \\
\hline $1981-2010$ & February & 5 & 35.2 & -0.12 & 0.04 & 0.8495 & A & NS \\
\hline 1951-1980 & February & 6 & 36.56 & & & & A & \\
\hline $1981-2010$ & February & 6 & 36.58 & 0.02 & 0.00 & 0.9735 & A & NS \\
\hline 1951-1980 & March & 7 & 36.97 & & & & A & \\
\hline $1981-2010$ & March & 7 & 37.03 & 0.06 & 0.02 & 0.9000 & A & NS \\
\hline $1951-1980$ & March & 8 & 38.3 & & & & A & \\
\hline $1981-2010$ & March & 8 & 37.7 & -0.6 & 1.25 & 0.2675 & A & NS \\
\hline 1951-1980 & March & 9 & 39 & & & & A & \\
\hline $1981-2010$ & March & 9 & 39.1 & 0.1 & 0.05 & 0.8190 & $A$ & NS \\
\hline $1951-1980$ & April & 10 & 39.9 & & & & A & \\
\hline $1981-2010$ & April & 10 & 40.69 & 0.79 & 3.29 & 0.0750 & A & NS \\
\hline $1951-1980$ & April & 11 & 40.3 & & & & A & \\
\hline $1981-2010$ & April & 11 & 41.3 & 1 & 3.61 & 0.0629 & A & NS \\
\hline 1951-1980 & April & 12 & 41.1 & & & & A & \\
\hline $1981-2010$ & April & 12 & 41.6 & 0.5 & 1.66 & 0.2028 & A & NS \\
\hline $1951-1980$ & May & 13 & 41.3 & & & & A & \\
\hline $1981-2010$ & May & 13 & 41.9 & 0.6 & 3.20 & 0.0792 & A & NS \\
\hline 1951-1980 & May & 14 & 41.8 & & & & A & \\
\hline $1981-2010$ & May & 14 & 42.5 & 0.7 & 3.36 & 0.0721 & A & NS \\
\hline $1951-1980$ & May & 15 & 41.61 & & & & A & \\
\hline $1981-2010$ & May & 15 & 42 & 0.39 & 1.31 & 0.2568 & A & NS \\
\hline $1951-1980$ & June & 16 & 40.72 & & & & A & \\
\hline $1981-2010$ & June & 16 & 41.21 & 0.49 & 1.47 & 0.2311 & A & NS \\
\hline $1951-1980$ & June & 17 & 39.43 & & & & A & \\
\hline $1981-2010$ & June & 17 & 40.4 & 0.97 & 5.32 & 0.0248 & $B$ & $\mathrm{~s}$ \\
\hline $1951-1980$ & June & 18 & 37.93 & & & & A & \\
\hline 1981-2010 & June & 18 & 38.94 & 1.01 & 6.19 & 0.0159 & B & $\mathrm{s}$ \\
\hline
\end{tabular}

\begin{tabular}{|c|c|c|c|c|c|c|c|c|}
\hline Periods & Month & Decades & Means & Diff. $\left({ }^{\circ} \mathrm{C}\right)$ & F-ratio & P-value & H. G. & S.Level \\
\hline $1951-1980$ & July & 19 & $\quad 36.34$ & & & & A & \\
\hline $1981-2010$ & July & 19 & 37.28 & 0.94 & 6.49 & 0.0137 & B & $\mathrm{s}$ \\
\hline $1951-1980$ & July & 20 & 34.1 & & & & A & \\
\hline $1981-2010$ & July & 20 & 35.99 & 1.89 & 19.95 & 0.0000 & $B$ & $s$ \\
\hline $1951-1980$ & July & 21 & 33.03 & & & & A & \\
\hline $1981-2010$ & July & 21 & 34.4 & 1.37 & 12.89 & 0.0007 & B & $s$ \\
\hline $1951-1980$ & August & 22 & 32.46 & & & & A & \\
\hline 1981-2010 & August & 22 & 33.65 & 1.19 & 10.58 & 0.0020 & B & s \\
\hline $1951-1980$ & August & 23 & 32.34 & & & & A & \\
\hline $1981-2010$ & August & 23 & 33.44 & 1.1 & 6.66 & 0.0125 & B & $s$ \\
\hline $1951-1980$ & August & 24 & 32.17 & & & & A & \\
\hline $1981-2010$ & August & 24 & 33.3 & 1.13 & 8.43 & 0.0054 & $\begin{array}{l}4 . \\
\end{array}$ & $s$ \\
\hline 1951-1980 & September & 25 & 32.76 & & & & A & \\
\hline $1981-2010$ & September & 25 & 33.81 & 1.05 & 9.24 & 0.0036 & B & $\mathrm{s}$ \\
\hline $1951-1980$ & September & 26 & 33.74 & & & & A & \\
\hline $1981-2010$ & September & 26 & 35.08 & 1.34 & 11.62 & 0.0012 & B & $\mathrm{s}$ \\
\hline $1951-1980$ & September & 27 & 35.12 & & & & A & \\
\hline $1981-2010$ & September & 27 & 36.55 & 1.43 & 13.45 & 0.0006 & B & s \\
\hline 1951-1980 & October & 28 & 36.55 & & & & A & \\
\hline $1981-2010$ & October & 28 & 37.68 & 1.13 & 5.90 & 0.0184 & B & s \\
\hline $1951-1980$ & October & 29 & 37.41 & & & & A & \\
\hline $1981-2010$ & October & 29 & 38.49 & 1.08 & 9.86 & 0.0027 & B & $s$ \\
\hline 1951-1980 & October & 30 & 37.13 & & & & A & \\
\hline $1981-2010$ & October & 30 & 38.42 & 1.29 & 16.07 & 0.0002 & B & s \\
\hline 1951-1980 & November & 31 & 36.92 & & & & A & \\
\hline $1981-2010$ & November & 31 & 37.42 & 0.5 & 2.11 & 0.1525 & A & NS \\
\hline $1951-1980$ & November & 32 & 36.2 & & & & A & \\
\hline $1981-2010$ & November & 32 & 36.42 & 0.22 & 0.46 & 0.5015 & A & NS \\
\hline 1951-1980 & November & 33 & 34.74 & & & & A & \\
\hline $1981-2010$ & November & 33 & 35.19 & 0.45 & 0.66 & 0.4217 & 7 A & NS \\
\hline $1951-1980$ & December & 34 & 33.21 & & & & A & \\
\hline $1981-2010$ & December & 34 & 33.27 & 0.06 & 0.01 & 0.9162 & A & NS \\
\hline $1951-1980$ & December & 35 & 31.76 & & & & A & \\
\hline $1981-2010$ & December & 35 & 32.53 & 0.77 & 1.27 & 0.2644 & 4.A & NS \\
\hline $1951-1980$ & December & 36 & 30.74 & & & & A & \\
\hline $1981-2010$ & December & 36 & 31.73 & 0.99 & 2.62 & 0.1112 & A $\mathrm{A}$ & NS \\
\hline \multicolumn{3}{|c|}{ Average Periods $1951-1980$} & 36.1 & & & & A & \\
\hline \multicolumn{3}{|c|}{ Average Periods $1981-2010$} & 36.63 & 0.53 & 10.65 & 0.0019 & 9 & $\mathrm{~s}$ \\
\hline
\end{tabular}

NB: Diff= difference in average temperature between the two periods $(1951-1980)$ and $(1981-2010)$

$\mathrm{S}=$ significance; $\mathrm{NS}=$ not significance. 
Table 3. Minimum temperature every ten days statistical analysis of the period 1951-1980 (30 years) and compared to the period 1981-2010 (30 years) at the meteorological station of Nioro du Sahel $\left(15.14^{\circ} \mathrm{N}\right.$ latitude; $9.36^{\circ} \mathrm{O}$; altitude $236 \mathrm{~m}$ ) in Mali

\begin{tabular}{|c|c|c|c|c|c|c|c|c|}
\hline Periods & Month & Decades & Means & Diff. $\left({ }^{\circ} \mathrm{C}\right)$ & F-ratio & P-value & H. G. & S.Leve \\
\hline $1951-1980$ & January & 1 & 13.17 & & & & A & \\
\hline $1981-2010$ & January & 1 & 13.35 & 0.18 & 0.06 & 0.8043 & A & NS \\
\hline $1951-1980$ & January & 2 & 13.2 & & & & $A$ & \\
\hline $1981-2010$ & January & 2 & 13.5 & 0.30 & 0.19 & 0.6633 & $A$ & NS \\
\hline $1951-1980$ & January & 3 & 13.33 & & & & A & \\
\hline $1981-2010$ & January & 3 & 14.43 & 1.10 & 2.48 & 0.1213 & A & NS \\
\hline $1951-1980$ & February & 4 & 14.57 & & & & $A$ & \\
\hline $1981-2010$ & February & 4 & 15.91 & 1.34 & 4.26 & 0.0438 & $B$ & $\mathrm{~s}$ \\
\hline $1951-1980$ & February & 5 & 15.62 & & & & A & \\
\hline $1981-2010$ & February & 5 & 17.38 & 1.76 & 6.66 & 0.0126 & B & $\mathrm{s}$ \\
\hline 1951-1980 & February & 6 & 16.44 & & & & A & \\
\hline $1981-2010$ & February & 6 & 18.17 & 1.73 & 9.58 & 0.0031 & B & $\mathrm{s}$ \\
\hline $1951-1980$ & March & 7 & 17.17 & & & & A & \\
\hline $1981-2010$ & March & 7 & 19.19 & 2.02 & 11.29 & 0.0014 & B & $\mathrm{s}$ \\
\hline $1951-1980$ & March & 8 & 18.73 & & & & A & \\
\hline $1981-2010$ & March & 8 & 20.11 & 1.38 & 4.84 & 0.0320 & $B$ & s \\
\hline $1951-1980$ & March & 9 & 19.94 & & & & A & \\
\hline $1981-2010$ & March & 9 & 21.92 & 1.98 & 8.43 & 0.0053 & B & $\mathrm{s}$ \\
\hline $1951-1980$ & April & 10 & 21.29 & & & & A & \\
\hline $1981-2010$ & April & 10 & 22.97 & 1.68 & 9.02 & 0.0040 & B & $\mathrm{s}$ \\
\hline $1951-1980$ & April & 11 & 22.47 & & & & A & \\
\hline $1981-2010$ & April & 11 & 24.65 & 2.18 & 11.23 & 0.0015 & B & $\mathrm{s}$ \\
\hline $1951-1980$ & April & 12 & 24.53 & & & & A & \\
\hline $1981-2010$ & April & 12 & 25.58 & 1.05 & 3.53 & 0.0657 & A & NS \\
\hline $1951-1980$ & May & 13 & 25.89 & & & & A & \\
\hline $1981-2010$ & May & 13 & 26.64 & 0.75 & 1.95 & $0.0700]$ & $A$ & NS \\
\hline $1951-1980$ & May & 14 & 26.56 & & & & A & \\
\hline $1981-2010$ & May & 14 & 27.76 & 1.20 & 7.03 & 0.0145 & B & $\mathrm{s}$ \\
\hline $1951-1980$ & May & 15 & 27.57 & & & & A & \\
\hline $1981-2010$ & May & 15 & 28.56 & 0.99 & 5.52 & 0.0224 & $B$ & $\mathrm{~s}$ \\
\hline $1951-1980$ & June & 16 & 27.2 & & & & A & \\
\hline $1981-2010$ & June & 16 & 27.9 & 0.70 & 3.30 & 0.0749 & $A$ & NS \\
\hline $1951-1980$ & June & 17 & 26.72 & & & & A & \\
\hline $1981-2010$ & June & 17 & 27.37 & 0.65 & 2.06 & 0.1569 & A & NS \\
\hline $1951-1980$ & June & 18 & 25.84 & & & & A & \\
\hline $1981-2010$ & June & 18 & 26.52 & 0.68 & 2.71 & 0.1051 & $A$ & NS \\
\hline
\end{tabular}

\begin{tabular}{|c|c|c|c|c|c|c|c|c|}
\hline Periods & Month & Decades & Means & Diff. $\left({ }^{\circ} \mathrm{C}\right)$ & F-ratio & P-value & H. G. & S.Leve \\
\hline 1951-1980 & July & 19 & 25.09 & \multirow[b]{2}{*}{0.55} & \multirow[b]{2}{*}{2.13} & \multirow{2}{*}{0.1497} & A & \multirow[b]{2}{*}{ NS } \\
\hline $1981-2010$ & July & 19 & 25.64 & & & & A & \\
\hline 1951-1980 & July & 20 & 24 & \multirow[b]{2}{*}{1} & \multirow[b]{2}{*}{7.69} & \multirow{2}{*}{0.0076} & A & \multirow[b]{2}{*}{ NS } \\
\hline $1981-2010$ & July & 20 & 25 & & & & A & \\
\hline $1951-1980$ & July & 21 & 23.64 & \multirow[b]{2}{*}{0.34} & \multirow[b]{2}{*}{0.98} & \multirow{2}{*}{0.3300} & A & \multirow[b]{2}{*}{ NS } \\
\hline 1981-2010 & July & 21 & 23.98 & & & & A & \\
\hline $1951-1980$ & August & 22 & 23.31 & \multirow[b]{2}{*}{0.45} & \multirow[b]{2}{*}{1.78} & \multirow{2}{*}{0.1872} & A & \multirow[b]{2}{*}{ NS } \\
\hline $1981-2010$ & August & 22 & 23.76 & & & & A & \\
\hline $1951-1980$ & August & 23 & 23.36 & \multirow[b]{2}{*}{0.04} & \multirow[b]{2}{*}{0.01} & \multirow{2}{*}{0.9174} & A & \multirow[b]{2}{*}{ NS } \\
\hline 1981-2010 & August & 23 & 23.4 & & & & A & \\
\hline 1951-1980 & August & 24 & 23 & \multirow[b]{2}{*}{0.18} & \multirow[b]{2}{*}{0.29} & \multirow{2}{*}{0.5914} & A & \multirow[b]{2}{*}{ NS } \\
\hline $1981-2010$ & August & 24 & 23.18 & & & & A & \\
\hline $1951-1980$ & September & 25 & 22.99 & \multirow[b]{2}{*}{0.12} & & & A & \\
\hline $1981-2010$ & September & 25 & 23.11 & & 0.14 & 0.7100 & A & NS \\
\hline $1951-1980$ & September & 26 & 23.23 & & & & A & \\
\hline 1981-2010 & September & 26 & 23.43 & 0.2 & 0.47 & 0.4967 & A & NS \\
\hline 1951-1980 & September & 27 & 23.51 & & & & A & \\
\hline $1981-2010$ & September & 27 & 23.55 & 0.04 & 0.01 & 0.9243 & A & NS \\
\hline $1951-1980$ & October & 28 & 23.26 & & & & A & \\
\hline $1981-2010$ & October & 28 & 23.86 & 0.6 & 2.57 & 0.1147 & A & NS \\
\hline $1951-1980$ & October & 29 & 22.97 & & & & A & \\
\hline $1981-2010$ & October & 29 & 23.25 & 0.28 & 0.65 & 0.4241 & $A$ & NS \\
\hline $1951-1980$ & October & 30 & 21 & & & & A & \\
\hline $1981-2010$ & October & 30 & 21.44 & 0.44 & 0.80 & 0.3585 & A & NS \\
\hline 1951-1980 & November & 31 & 19.63 & & & & A & \\
\hline 1981-2010 & November & 31 & 19.83 & 0.2 & 0.17 & 0.6826 & A & NS \\
\hline 1951-1980 & November & 32 & 17.9 & & & & A & \\
\hline $1981-2010$ & November & 32 & 18.71 & 0.81 & 2.05 & 0.1577 & A & NS \\
\hline 1951-1980 & November & 33 & 16.41 & & & & A & \\
\hline $1981-2010$ & November & 33 & 16.67 & 0.26 & 0.19 & 0.6612 & A & NS \\
\hline $1951-1980$ & December & 34 & 14.9 & & & & A & \\
\hline $1981-2010$ & December & 34 & 15.8 & 0.9 & 2.07 & 0.1559 & A & NS \\
\hline $1951-1980$ & December & 35 & 14.1 & & & & A & \\
\hline $1981-2010$ & December & 35 & 14.91 & 0.81 & 1.64 & 0.2056 & A & NS \\
\hline $1951-1980$ & December & 36 & 12.87 & & & & A & \\
\hline $1981-2010$ & December & 36 & 13.75 & 0.88 & 2.28 & 0.1364 & A & NS \\
\hline Aver & ge Periods 19 & 1980 & 20.71 & & & & A & \\
\hline Aver & ge Periods 19 & 2010 & 21.53 & 0.82 & 10.02 & 0.0025 & $B$ & $\mathrm{~s}$ \\
\hline
\end{tabular}


Table 4. Maximum temperature every ten days statistical analysis of the period 1951-1980 (30 years) and compared to the period 1981-2010 (30 years) at the meteorological station of Segou in Mali. (13.24 ${ }^{\circ} \mathrm{N}$ latitude; $6.09^{\circ} \mathrm{O}$ longitude, altitude $\left.288 \mathrm{~m}\right)$

\begin{tabular}{|c|c|c|c|c|c|c|c|c|}
\hline Periods & Month & Decades & Means $\left({ }^{\circ} \mathrm{C}\right)$ & Diff. $\left({ }^{\circ} \mathrm{C}\right)$ & F-ratio & P-value & H. G. & S.Level \\
\hline $1951-1980$ & January & 1 & 31.88 & \multirow[b]{2}{*}{0.4} & \multirow[b]{2}{*}{0.41} & \multirow{2}{*}{0.5228} & A & \multirow[b]{2}{*}{ NS } \\
\hline $1981-2010$ & January & 1 & 32.28 & & & & A & \\
\hline 1951-1980 & January & 2 & 31.92 & \multirow[b]{2}{*}{0.83} & \multirow[b]{2}{*}{1.52} & \multirow[b]{2}{*}{0.2219} & A & \multirow[b]{2}{*}{ NS } \\
\hline $1981-2010$ & January & 2 & 32.75 & & & & $A$ & \\
\hline $1951-1980$ & January & 3 & 32.87 & \multirow[b]{2}{*}{0.74} & \multirow[b]{2}{*}{1.05} & \multirow[b]{2}{*}{0.3110} & A & \multirow[b]{2}{*}{ NS } \\
\hline $1981-2010$ & January & 3 & 33.61 & & & & A & \\
\hline $1951-1980$ & February & 4 & 34.01 & \multirow[b]{2}{*}{1.39} & \multirow[b]{2}{*}{4.91} & \multirow[b]{2}{*}{0.0307} & A & \multirow[b]{2}{*}{ s } \\
\hline $1981-2010$ & February & 4 & 35.4 & & & & $B$ & \\
\hline $1951-1980$ & February & 5 & 35.53 & \multirow[b]{2}{*}{0.88} & \multirow[b]{2}{*}{3.74} & \multirow[b]{2}{*}{0.0581} & A & \multirow[b]{2}{*}{ NS } \\
\hline $1981-2010$ & February & 5 & 36.41 & & & & $A$ & \\
\hline 1951-1980 & February & 6 & 36.44 & \multirow[b]{2}{*}{1.29} & \multirow[b]{2}{*}{6.07} & \multirow[b]{2}{*}{0.0167} & A & \multirow[b]{2}{*}{ s } \\
\hline $1981-2010$ & February & 6 & 37.73 & & & & $B$ & \\
\hline $1951-1980$ & March & 7 & 36.75 & \multirow[b]{2}{*}{1.51} & \multirow[b]{2}{*}{11.12} & & A & \\
\hline $1981-2010$ & March & 7 & 38.26 & & & 0.0015 & $B$ & S \\
\hline $1951-1980$ & March & 8 & 37.99 & & & & A & \\
\hline $1981-2010$ & March & 8 & 38.88 & 0.89 & 3.94 & 0.0520 & $A$ & NS \\
\hline $1951-1980$ & March & 9 & 38.35 & & & & A & \\
\hline $1981-2010$ & March & 9 & 40.22 & 1.87 & 18.00 & 0.0001 & $B$ & S \\
\hline $1951-1980$ & April & 10 & 38.85 & & & & A & \\
\hline $1981-2010$ & April & 10 & 41.03 & 2.18 & 34.51 & 0.0000 & $B$ & s \\
\hline $1951-1980$ & April & 11 & 39.22 & & & & A & \\
\hline $1981-2010$ & April & 11 & 41.29 & 2.07 & 31.73 & 0.0000 & $B$ & $\mathrm{~s}$ \\
\hline $1951-1980$ & April & 12 & 39.27 & & & & A & \\
\hline $1981-2010$ & April & 12 & 40.88 & 1.61 & 19.91 & 0.0000 & $B$ & S \\
\hline $1951-1980$ & May & 13 & 39.11 & & & & A & \\
\hline $1981-2010$ & May & 13 & 41.08 & 1.97 & 25.18 & 0.0000 & $B$ & $s$ \\
\hline 1951-1980 & May & 14 & 39.15 & & & & A & \\
\hline $1981-2010$ & May & 14 & 40.85 & 1.7 & 25.72 & 0.0000 & $B$ & S \\
\hline $1951-1980$ & May & 15 & 38.3 & & & & A & \\
\hline $1981-2010$ & May & 15 & 39.75 & 1.45 & 12.09 & 0.0010 & $B$ & s \\
\hline $1951-1980$ & June & 16 & 37.23 & & & & A & \\
\hline $1981-2010$ & June & 16 & 38.57 & 1.34 & 8.37 & 0.0054 & $B$ & $\mathrm{~s}$ \\
\hline $1951-1980$ & June & 17 & 35.92 & & & & A & \\
\hline $1981-2010$ & June & 17 & 37.71 & 1.79 & 14.48 & 0.0003 & $B$ & s \\
\hline 1951-1980 & June & 18 & 34.77 & & & & A & \\
\hline $1981-2010$ & June & 18 & 36.01 & 1.24 & 11.35 & 0.0013 & $B$ & $\mathrm{~s}$ \\
\hline
\end{tabular}

\begin{tabular}{|c|c|c|c|c|c|c|c|c|}
\hline Periods & Month & Decades & Means $\left({ }^{\circ} \mathrm{C}\right)$ & Diff. $\left({ }^{\circ} \mathrm{C}\right)$ & F-ratio & P-value & H. G. & S.Level \\
\hline 1951-1980 & July & 19 & 33.58 & & & & A & \\
\hline $1981-2010$ & July & 19 & 34.79 & 1.21 & 10.69 & 0.0000 & $B$ & $s$ \\
\hline 1951-1980 & July & 20 & 32.14 & & & & A & \\
\hline $1981-2010$ & July & 20 & 33.5 & 1.36 & 17.34 & 0.0000 & B $B$ & $\mathrm{~s}$ \\
\hline 1951-1980 & July & 21 & 31.14 & & & & A & \\
\hline $1981-2010$ & July & 21 & 32.66 & 1.52 & 23.23 & 0.0000 & $B$ & $\mathrm{~s}$ \\
\hline $1951-1980$ & August & $\begin{array}{r}22 \\
-- \\
\end{array}$ & $\begin{array}{r}30.88 \\
\end{array}$ & & & & A & \\
\hline $1981-2010$ & August & 22 & 32.02 & 1.14 & 21.63 & $\begin{array}{r}0.0109 \\
-\end{array}$ & B & $\mathrm{s}$ \\
\hline 1951-1980 & August & 23 & 30.51 & & & & A & \\
\hline $1981-2010$ & August & 23 & 31.95 & 1.44 & 30.09 & 0.0132 & $B$ & $s$ \\
\hline $1951-1980$ & August & 24 & 30.49 & & & & A & \\
\hline $1981-2010$ & August & 24 & 32.1 & 1.61 & 40.77 & 0.0009 & $B$ & $\mathrm{~s}$ \\
\hline 1951-1980 & September & 25 & 30.86 & & & & A & \\
\hline 1981-2010 & September & 25 & 32.07 & 1.21 & 22.41 & 0.0000 & B & $\mathrm{s}$ \\
\hline 1951-1980 & September & _.26 & 31.55 & & & & A & \\
\hline $1981-2010$ & September & 26 & 33.19 & 1.64 & 31.87 & 0.0000 & $B$ & $s$ \\
\hline $1951-1980$ & September & 27 & 32.66 & & & & A & \\
\hline $1981-2010$ & September & 27 & 34.6 & 1.94 & 29.80 & 0.0000 & B & $\mathrm{s}$ \\
\hline $1951-1980$ & October & $\begin{array}{r}28 \\
-\end{array}$ & 33.92 & & & & A & \\
\hline $1981-2010$ & October & 28 & 35.45 & 1.53 & 6.93 & 0.0109 & B & $\mathrm{s}$ \\
\hline 1951-1980 & October & 29 & 34.91 & & & & A & \\
\hline $1981-2010$ & October & 29 & 36.46 & 1.55 & 6.54 & 0.0132 & $B$ & $\mathrm{~s}$ \\
\hline $1951-1980$ & October & $\begin{array}{r}30 \\
- \\
-\end{array}$ & 34.96 & & & & A & \\
\hline $1981-2010$ & October & 30 & 37.13 & 2.17 & 12.36 & 0.0009 & B & $\mathrm{s}$ \\
\hline 1951-1980 & November & 31 & 35.22 & & & & A & \\
\hline 1981-2010 & November & 31 & 37.45 & 2.23 & 50.67 & 0.0000 & $B$ & $\mathrm{~s}$ \\
\hline $1951-1980$ & November & 32 & 35.04 & & & & A & \\
\hline $1981-2010$ & November & 32 & 37.01 & 1.97 & 39.50 & 0.0000 & B & $\mathrm{s}$ \\
\hline $1951-1980$ & November & 33 & 34.01 & & & & A & \\
\hline $1981-2010$ & November & 33 & 36.45 & 2.44 & 30.43 & 0.0000 & $B$ & $\mathrm{~s}$ \\
\hline $1951-1980$ & December & 34 & 32.86 & & & & A & \\
\hline $1981-2010$ & December & 34 & 34.85 & 1.99 & 14.68 & 0.0003 & B & $\mathrm{s}$ \\
\hline $1951-1980$ & December & 35 & 31.71 & & & & A & \\
\hline $1981-2010$ & December & 35 & 33.97 & 2.26 & 12.55 & 0.0008 & B & $\mathrm{s}$ \\
\hline $1951-1980$ & December & $\begin{array}{r}36 \\
- \\
-\end{array}$ & 31.15 & & & & A & \\
\hline $1981-2010$ & December & 36 & 33.54 & 2.39 & 14.80 & 0.0003 & B & $\mathrm{s}$ \\
\hline \multicolumn{3}{|c|}{ Average Periods 1951-1980 } & $\begin{array}{l}34.59 \\
\text { numanumumam }\end{array}$ & & & & $A$ & \\
\hline \multicolumn{3}{|c|}{ Average Periods 1981-2010 } & 36.16 & 1.57 & 64.15 & 0.0000 & $B$ & $\mathrm{~s}$ \\
\hline
\end{tabular}


Table 5. Minimum temperature every ten days statistical analysis of the period 1951-1980 (30 years) and compared to the period 1981-2010 (30 years) at the meteorological station of Segou in Mali. $\left(13.24^{\circ} \mathrm{N}\right.$ latitude, $6.09^{\circ} \mathrm{O}$ longitude; altitude $\left.288 \mathrm{~m}\right)$

\begin{tabular}{|c|c|c|c|c|c|c|c|c|}
\hline Periods & Month & Decades & Means $\left({ }^{\circ} \mathrm{C}\right)$ & Diff. $\left({ }^{\circ} \mathrm{C}\right)$ & F-ratio & P-value & H. G. & S.Level \\
\hline $1951-1980$ & January & 1 & 15.74 & & & & A & \\
\hline $1981-2010$ & January & 1 & 16.66 & 0.92 & 3.79 & 0.0563 & A & NS \\
\hline $1951-1980$ & January & 2 & 16 & & & & A & \\
\hline $1981-2010$ & January & 2 & 17.1 & 1.1 & 6.13 & 0.0162 & $B$ & s \\
\hline $1951-1980$ & January & 3 & 16.46 & & & & A & \\
\hline $1981-2010$ & January & 3 & 17.65 & 1.19 & 6.75 & 0.0119 & $B$ & $s$ \\
\hline $1951-1980$ & February & 4 & 17.6 & & & & A & \\
\hline $1981-2010$ & February & 4 & 19.03 & 1.43 & 10.04 & 0.0024 & $\mathrm{~B}$ & s \\
\hline $1951-1980$ & February & 5 & 18.62 & & & & A & \\
\hline $1981-2010$ & February & 5 & 20.29 & 1.67 & 15.01 & 0.0003 & $B$ & s \\
\hline $1951-1980$ & February & 6 & 19.47 & & & & A & \\
\hline $1981-2010$ & February & 6 & 21.2 & 1.73 & 22.16 & 0.0000 & B & s \\
\hline $1951-1980$ & March & 7 & 20.18 & & & & A & \\
\hline $1981-2010$ & March & 7 & 22.42 & 2.24 & 36.76 & 0.0000 & B & s \\
\hline 1951-1980 & March & 8 & 21.5 & & & & A & \\
\hline $1981-2010$ & March & 8 & 23.48 & 1.98 & 20.41 & 0.0000 & B & s \\
\hline 1951-1980 & March & 9 & 22.56 & & & & A & \\
\hline $1981-2010$ & March & 9 & 24.25 & 1.69 & 15.55 & 0.0002 & B & $\mathrm{s}$ \\
\hline $1951-1980$ & April & 10 & 23.39 & & & & A & \\
\hline $1981-2010$ & April & 10 & 25.5 & 2.11 & 38.48 & 0.0000 & B & s \\
\hline $1951-1980$ & April & 11 & 24.35 & & & & A & \\
\hline $1981-2010$ & April & 11 & 26.81 & 2.46 & 48.02 & 0.0000 & B & $\mathrm{s}$ \\
\hline $1951-1980$ & April & 12 & 25.29 & & & & $A$ & \\
\hline $1981-2010$ & April & 12 & 27.4 & 2.11 & 48.74 & 0.0000 & B & s \\
\hline $1951-1980$ & May & 13 & 25.66 & & & & A & \\
\hline $1981-2010$ & May & 13 & 27.5 & 1.84 & 35.94 & 0.0000 & B & s \\
\hline $1951-1980$ & May & 14 & 26 & & & & A & \\
\hline $1981-2010$ & May & 14 & 27.62 & 1.62 & 41.27 & 0.0000 & $B$ & $\mathrm{~s}$ \\
\hline $1951-1980$ & May & 15 & 25.86 & & & & A & \\
\hline $1981-2010$ & May & 15 & 27.27 & 1.41 & 43.22 & 0.0000 & B & s \\
\hline $1951-1980$ & June & 16 & 24.92 & & & & A & \\
\hline $1981-2010$ & June & 16 & 26.39 & 1.47 & 32.97 & 0.0000 & $B$ & $\mathrm{~s}$ \\
\hline $1951-1980$ & June & 17 & 24.44 & & & & A & \\
\hline $1981-2010$ & June & 17 & 25.94 & 1.5 & 23.54 & 0.0000 & B & $\mathrm{s}$ \\
\hline $1951-1980$ & June & 18 & 23.51 & & & & A & \\
\hline $1981-2010$ & June & 18 & 24.94 & 1.43 & 29.76 & 0.0000 & B & $\mathrm{s}$ \\
\hline
\end{tabular}

\begin{tabular}{|c|c|c|c|c|c|c|c|c|}
\hline Periods & Month & Decades & Means $\left({ }^{\circ} \mathrm{C}\right)$ & Diff. $\left({ }^{\circ} \mathrm{C}\right)$ & F-ratio & P-value & H. G. & S.Level \\
\hline $1951-1980$ & July & 19 & 23.19 & & & & A & \\
\hline $1981-2010$ & July & 19 & 24.3 & 1.11 & 22.60 & 0.0000 & $B$ & $\mathrm{~s}$ \\
\hline $1951-1980$ & July & 20 & 22.54 & & & & A & \\
\hline $1981-2010$ & July & 20 & 23.74 & 1.2 & 31.21 & 0.0000 & B & $\mathrm{s}$ \\
\hline 1951-1980 & July & 21 & 22.28 & & & & A & \\
\hline $1981-2010$ & July & 21 & 23.43 & 1.15 & 54.87 & 0.0000 & $B$ & s \\
\hline $1951-1980$ & August & 22 & 22.24 & & & & A & \\
\hline $1981-2010$ & August & 22 & 23.21 & 0.97 & 48.19 & 0.0000 & B & $\mathrm{s}$ \\
\hline $1951-1980$ & August & 23 & 22.19 & & & & A & \\
\hline $1981-2010$ & August & 23 & 23.12 & 0.93 & 46.00 & 0.0000 & $B$ & $\mathrm{~s}$ \\
\hline $1951-1980$ & August & 24 & 22.01 & & & & A & \\
\hline $1981-2010$ & August & 24 & 23 & 0.99 & 62.67 & 0.0000 & B & $\mathrm{s}$ \\
\hline $1951-1980$ & September & 25 & 21.88 & & & & A & \\
\hline $1981-2010$ & September & 25 & 22.96 & 1.08 & 62.10 & 0.0000 & $B$ & $\mathrm{~s}$ \\
\hline $1951-1980$ & September & 26 & 22 & & & & A & \\
\hline $1981-2010$ & September & 26 & 23.15 & 1.15 & 52.49 & 0.0000 & B & $\mathrm{s}$ \\
\hline 1951-1980 & September & 27 & 22.27 & & & & A & \\
\hline $1981-2010$ & September & 27 & 23.54 & 1.27 & 63.22 & 0.0000 & B & $\mathrm{s}$ \\
\hline $1951-1980$ & October & 28 & 22.25 & & & & A & \\
\hline $1981-2010$ & October & 28 & 23.69 & 1.44 & 65.01 & 0.0000 & $B$ & $\mathrm{~s}$ \\
\hline 1951-1980 & October & 29 & 22.01 & & & & A & \\
\hline $1981-2010$ & October & 29 & 23.74 & 1.73 & 56.31 & 0.0000 & B & $\mathrm{s}$ \\
\hline $1951-1980$ & October & 30 & 20.87 & & & & A & \\
\hline $1981-2010$ & October & 30 & 22.71 & 1.84 & 39.09 & 0.0000 & $B$ & $\mathrm{~s}$ \\
\hline $1951-1980$ & November & 31 & 19.84 & & & & A & \\
\hline $1981-2010$ & November & 31 & 21.37 & 1.53 & 25.62 & 0.0000 & B & $\mathrm{s}$ \\
\hline 1951-1980 & November & 32 & 18.93 & & & & A & \\
\hline $1981-2010$ & November & 32 & 20.64 & 1.71 & 32.47 & 0.0000 & B & $\mathrm{s}$ \\
\hline $1951-1980$ & November & 33 & 18.13 & & & & A & \\
\hline $1981-2010$ & November & 33 & 19.7 & 1.57 & 21.82 & 0.0000 & $B$ & $\mathrm{~s}$ \\
\hline 1951-1980 & December & 34 & 17.08 & & & & A & \\
\hline $1981-2010$ & December & 34 & 18.69 & 1.61 & 16.59 & 0.0001 & B & $\mathrm{s}$ \\
\hline $1951-1980$ & December & 35 & 16.32 & & & & A & \\
\hline $1981-2010$ & December & 35 & 17.8 & 1.48 & 11.91 & 0.0011 & B & $\mathrm{s}$ \\
\hline $1951-1980$ & December & 36 & 15.92 & & & & A & \\
\hline $1981-2010$ & December & 36 & 16.95 & 1.03 & 8.27 & 0.0056 & $B$ & $\mathrm{~s}$ \\
\hline \multicolumn{3}{|c|}{ Average Periods 1951-1980 } & 21.21 & & & & A & \\
\hline \multicolumn{3}{|c|}{ Average Periods 1981-2010 } & 22.7 & 1.49 & 115.32 & 0.0000 & $B$ & $\mathrm{~s}$ \\
\hline
\end{tabular}


Table 6. Maximum temperature every ten days statistical analysis of the period 1951-1980 (30 years) and compared to the period 1981-2010 (30 years) at the meteorological station of Sotuba in Mali $\left(12.39^{\circ} \mathrm{N}\right.$ latitude; $7.56^{\circ} \mathrm{O}$ longitude, altitude $\left.320 \mathrm{~m}\right)$

\begin{tabular}{|c|c|c|c|c|c|c|c|c|}
\hline Periods & Month & Decades & Means $\left({ }^{\circ} \mathrm{C}\right)$ & Diff. $\left({ }^{\circ} \mathrm{C}\right)$ & F-ratio & P-value & H. G. & S.Level \\
\hline $1951-1980$ & January & 1 & 33.2 & \multirow[b]{2}{*}{-1.46} & \multirow[b]{2}{*}{10.83} & \multirow[b]{2}{*}{0.0017} & A & \multirow[b]{2}{*}{ s } \\
\hline $1981-2010$ & January & 1 & 31.74 & & & & $\mathrm{~B}$ & \\
\hline $1951-1980$ & January & 2 & 33.3 & \multirow[b]{2}{*}{-1.1} & \multirow[b]{2}{*}{3.71} & \multirow{2}{*}{0.0589} & A & \multirow[b]{2}{*}{$\mathrm{s}$} \\
\hline $1981-2010$ & January & 2 & 32.2 & & & & $\mathrm{~B}$ & \\
\hline $1951-1980$ & January & 3 & 34.23 & \multirow[b]{2}{*}{-0.91} & \multirow[b]{2}{*}{2.55} & \multirow{2}{*}{0.1158} & A & \multirow[b]{2}{*}{ s } \\
\hline $1981-2010$ & January & 3 & 33.32 & & & & A & \\
\hline $1951-1980$ & February & 4 & 35.12 & \multirow[b]{2}{*}{-0.34} & \multirow[b]{2}{*}{0.45} & \multirow{2}{*}{0.5039} & A & \multirow[b]{2}{*}{ NS } \\
\hline $1981-2010$ & February & 4 & 34.78 & & & & $\mathrm{~A}$ & \\
\hline $1951-1980$ & February & 5 & 36.48 & \multirow[b]{2}{*}{-0.63} & \multirow[b]{2}{*}{2.53} & \multirow[b]{2}{*}{0.1173} & A & \multirow[b]{2}{*}{ NS } \\
\hline $1981-2010$ & February & 5 & 35.85 & & & & $\mathrm{~A}$ & \\
\hline $1951-1980$ & February & 6 & 37.25 & \multirow[b]{2}{*}{-0.05} & \multirow[b]{2}{*}{0.06} & \multirow{2}{*}{0.8143} & A & \multirow[b]{2}{*}{ NS } \\
\hline $1981-2010$ & February & 6 & 37.2 & & & & $\mathrm{~A}$ & \\
\hline $1951-1980$ & March & 7 & 37.44 & \multirow[b]{2}{*}{0.44} & \multirow[b]{2}{*}{1.71} & & A & \\
\hline $1981-2010$ & March & 7 & 37.88 & & & 0.1962 & $\mathrm{~A}$ & NS \\
\hline 1951-1980 & March & 8 & 38.44 & & & & A & \\
\hline $1981-2010$ & March & 8 & 38.35 & -0.09 & 0.07 & 0.7875 & $\mathrm{~A}$ & NS \\
\hline $1951-1980$ & March & 9 & 38.61 & & & & A & \\
\hline $1981-2010$ & March & 9 & 39.06 & 0.45 & 1.83 & 0.1819 & A & NS \\
\hline $1951-1980$ & April & 10 & 38.8 & & & & A & \\
\hline $1981-2010$ & April & 10 & 39.71 & 0.91 & 7.37 & 0.0087 & $\mathrm{~B}$ & s \\
\hline $1951-1980$ & April & 11 & 39.03 & & & & A & \\
\hline $1981-2010$ & April & 11 & 39.87 & 0.84 & 6.05 & 0.0169 & B & $s$ \\
\hline 1951-1980 & April & 12 & 38.55 & & & & A & \\
\hline $1981-2010$ & April & 12 & 39.35 & 0.8 & 1.04 & 0.3072 & $\mathrm{~A}$ & NS \\
\hline $1951-1980$ & May & 13 & 38.32 & & & & A & \\
\hline $1981-2010$ & May & 13 & 39.31 & 0.99 & 8.23 & 0.0057 & B & $s$ \\
\hline $1951-1980$ & May & 14 & 38.17 & & & & A & \\
\hline $1981-2010$ & May & 14 & 38.65 & 0.48 & 1.94 & 0.1700 & $\mathrm{~A}$ & NS \\
\hline $1951-1980$ & May & 15 & 37.09 & & & & A & \\
\hline $1981-2010$ & May & 15 & 37.71 & 0.62 & 2.19 & 0.1400 & $\mathrm{~A}$ & NS \\
\hline $1951-1980$ & June & 16 & 35.82 & & & & A & \\
\hline $1981-2010$ & June & 16 & 36.42 & 0.6 & 1.73 & 0.1930 & A & NS \\
\hline $1951-1980$ & June & 17 & 34.39 & & & & A & \\
\hline $1981-2010$ & June & 17 & 35.85 & 1.46 & 17.35 & 0.0001 & B & $s$ \\
\hline $1951-1980$ & June & 18 & 33.45 & & & & A & \\
\hline $1981-2010$ & June & 18 & 34.18 & 0.73 & 5.74 & 0.0198 & $\mathrm{~B}$ & $\mathrm{~s}$ \\
\hline
\end{tabular}

\begin{tabular}{|c|c|c|c|c|c|c|c|c|}
\hline Periods & Month & Decades & Means $\left({ }^{\circ} \mathrm{C}\right)$ & Diff. $\left({ }^{\circ} \mathrm{C}\right)$ & F-ratio & P-value & H. G. & S.Level \\
\hline 1951-1980 & July & 19 & 32.1 & \multirow[b]{2}{*}{0.81} & \multirow[b]{2}{*}{5.51} & \multirow{2}{*}{0.0224} & A & \multirow[b]{2}{*}{$\mathrm{s}$} \\
\hline $1981-2010$ & July & 19 & 32.91 & & & & B & \\
\hline 1951-1980 & July & 20 & 31.24 & \multirow[b]{2}{*}{0.56} & \multirow[b]{2}{*}{4.21} & \multirow[b]{2}{*}{0.0448} & A & \multirow[b]{2}{*}{ s } \\
\hline $1981-2010$ & July & 20 & 31.8 & & & & B & \\
\hline $1951-1980$ & July & 21 & 30.39 & \multirow[b]{2}{*}{0.61} & \multirow[b]{2}{*}{4.26} & \multirow[b]{2}{*}{0.0434} & A & \multirow[b]{2}{*}{ s } \\
\hline $1981-2010$ & July & 21 & 31 & & & & B & \\
\hline $1951-1980$ & August & 22 & 30.34 & \multirow[b]{2}{*}{0.47} & \multirow[b]{2}{*}{4.09} & \multirow{2}{*}{4.0477} & A & \multirow[b]{2}{*}{$s$} \\
\hline $1981-2010$ & August & 22 & 30.81 & & & & $\mathrm{~B}$ & \\
\hline $1951-1980$ & August & 23 & 29.97 & \multirow[b]{2}{*}{1.06} & \multirow[b]{2}{*}{11.11} & \multirow[b]{2}{*}{0.0015} & A & \multirow[b]{2}{*}{$s$} \\
\hline $1981-2010$ & August & 23 & 31.03 & & & & B & \\
\hline 1951-1980 & August & 24 & 30.22 & \multirow[b]{2}{*}{0.92} & \multirow[b]{2}{*}{13.08} & \multirow[b]{2}{*}{0.0006} & A & \multirow[b]{2}{*}{$s$} \\
\hline $1981-2010$ & August & 24 & 31.14 & & & & B & \\
\hline $1951-1980$ & September & 25 & 30.63 & \multirow[b]{2}{*}{0.7} & & & A & \\
\hline $1981-2010$ & September & 25 & 31.33 & & 7.35 & 0.0088 & $B$ & $s$ \\
\hline $1951-1980$ & September & 26 & 31.31 & & & & A & \\
\hline $1981-2010$ & September & 26 & 32.13 & 0.82 & 12.08 & 0.0010 & $B$ & s \\
\hline $1951-1980$ & September & 27 & 32.1 & & & & A & \\
\hline $1981-2010$ & September & 27 & 33 & 0.9 & 10.92 & 0.0016 & B & $s$ \\
\hline 1951-1980 & October & 28 & 32.95 & & & & A & \\
\hline $1981-2010$ & October & 28 & 33.84 & 0.89 & 6.37 & 0.0143 & $B$ & s \\
\hline $1951-1980$ & October & 29 & $\begin{array}{r}33.8 \\
\end{array}$ & & & & A & \\
\hline $1981-2010$ & October & 29 & 34.62 & 0.82 & 6.14 & 0.0162 & B & $\mathrm{s}$ \\
\hline $1951-1980$ & October & 30 & 33.93 & & & & A & \\
\hline $1981-2010$ & October & 30 & 35.1 & 1.17 & 13.32 & 0.0006 & B & s \\
\hline $1951-1980$ & November & 31 & 34.59 & & & & A & \\
\hline $1981-2010$ & November & 31 & 35.33 & 0.74 & 5.41 & 0.0235 & $B$ & $s$ \\
\hline 1951-1980 & November & 32 & 34.65 & & & & A & \\
\hline $1981-2010$ & November & 32 & 35.14 & 0.49 & 1.91 & 0.1725 & $\mathrm{~A}$ & NS \\
\hline $1951-1980$ & November & 33 & 34.02 & & & & A & \\
\hline $1981-2010$ & November & 33 & 35.02 & 1 & 9.05 & 0.0039 & $B$ & s \\
\hline $1951-1980$ & December & 34 & 33.43 & & & & A & \\
\hline $1981-2010$ & December & 34 & 33.73 & 0.3 & 1.06 & 0.3083 & A & NS \\
\hline $1951-1980$ & December & 35 & 32.64 & & & & A & \\
\hline $1981-2010$ & December & 35 & 32.85 & 0.21 & 0.21 & 0.6453 & $\mathrm{~A}$ & NS \\
\hline $1951-1980$ & December & 36 & 32.39 & & & & A & \\
\hline $1981-2010$ & December & 36 & 32.47 & 0.08 & 0.02 & 0.8797 & $\mathrm{~A}$ & NS \\
\hline Aver & ge Periods 19 & 1980 & 34.41 & & & & A & \\
\hline Aver & ge Periods 19 & 2010 & 34.85 & 0.44 & 9.35 & 0.0034 & $\mathrm{~B}$ & $\mathrm{~s}$ \\
\hline
\end{tabular}


Table 7. Minimum temperature every ten days statistical analysis of the period 1951-1980 (30 years) and compared to the period 1981-2010 (30 years) at the meteorological station of Sotuba in Mali. $\left(12.39^{\circ} \mathrm{N}\right.$ latitude, $7.56^{\circ} \mathrm{O}$ longitude, altitude $\left.320 \mathrm{~m}\right)$

\begin{tabular}{|c|c|c|c|c|c|c|c|c|}
\hline Periods & Month & Decades & Means $\left({ }^{\circ} \mathrm{C}\right)$ & Diff. $\left({ }^{\circ} \mathrm{C}\right)$ & F-ratio & P-value & H. G. & S.Leve \\
\hline $1951-1980$ & January & 1 & 13.76 & & & & A & \\
\hline $1981-2010$ & January & 1 & 15.38 & 1.62 & 10.00 & 0.0025 & $B$ & $\mathrm{~s}$ \\
\hline $1951-1980$ & January & 2 & 14.1 & & & & A & \\
\hline $1981-2010$ & January & 2 & 16.12 & 2.02 & 15.08 & 0.0003 & B & s \\
\hline $1951-1980$ & January & 3 & 14.66 & & & & A & \\
\hline $1981-2010$ & January & 3 & 16.67 & 2.01 & 13.93 & 0.0004 & B & $\mathrm{s}$ \\
\hline 1951-1980 & February & 4 & 15.86 & & & & A & \\
\hline $1981-2010$ & February & 4 & 17.92 & 2.06 & 15.67 & 0.0002 & $B$ & $\mathrm{~s}$ \\
\hline $1951-1980$ & February & 5 & 17.21 & & & & A & \\
\hline $1981-2010$ & February & 5 & 19.36 & 2.15 & 13.68 & 0.0005 & B & $\mathrm{s}$ \\
\hline 1951-1980 & February & 6 & 17.99 & & & & A & \\
\hline $1981-2010$ & February & 6 & 20.41 & 2.42 & 21.47 & 0.0000 & $B$ & $s$ \\
\hline $1951-1980$ & March & 7 & 18.95 & & & & A & \\
\hline $1981-2010$ & March & 7 & 21.56 & 2.61 & 30.61 & 0.0000 & B & $\mathrm{s}$ \\
\hline $1951-1980$ & March & 8 & 20.25 & & & & A & \\
\hline $1981-2010$ & March & 8 & 22.28 & 2.03 & 18.67 & 0.0001 & $B$ & $s$ \\
\hline $1951-1980$ & March & 9 & 21.25 & & & & A & \\
\hline $1981-2010$ & March & 9 & 22.97 & 1.72 & 11.46 & 0.0013 & $B$ & s \\
\hline $1951-1980$ & April & 10 & 21.97 & & & & A & \\
\hline $1981-2010$ & April & 10 & 23.88 & 1.91 & 12.53 & 0.0008 & $B$ & $s$ \\
\hline 1951-1980 & April & 11 & 23.15 & & & & A & \\
\hline $1981-2010$ & April & 11 & 24.93 & 1.78 & 9.02 & 0.0039 & $B$ & $\mathrm{~s}$ \\
\hline $1951-1980$ & April & 12 & 4.33 & & & & A & \\
\hline $1981-2010$ & April & 12 & 25.39 & 21.06 & 5.47 & 0.0228 & $B$ & $\mathrm{~s}$ \\
\hline $1951-1980$ & May & 13 & 24.2 & & & & A & \\
\hline $1981-2010$ & May & 13 & 25.65 & 1.45 & 13.02 & 0.0006 & B & $s$ \\
\hline 1951-1980 & May & 14 & 24.63 & & & & A & \\
\hline $1981-2010$ & May & 14 & 25.27 & 0.64 & 3.79 & 0.0564 & $A$ & NS \\
\hline $1951-1980$ & May & 15 & 24.17 & & & & A & \\
\hline $1981-2010$ & May & 15 & 24.85 & 0.68 & 3.78 & 0.0567 & A & NS \\
\hline $1951-1980$ & June & 16 & 23.53 & & & & A & \\
\hline $1981-2010$ & June & 16 & 24.21 & 0.68 & 3.75 & 0.0058 & $\bar{A}$ & NS \\
\hline $1951-1980$ & June & 17 & 22.96 & & & & A & \\
\hline $1981-2010$ & June & 17 & 23.49 & 0.53 & 1.55 & 0.2180 & $A$ & NS \\
\hline $1951-1980$ & June & 18 & 22.29 & & & & A & \\
\hline 1981-2010 & June & 18 & 22.65 & 0.36 & 0.95 & 0.3300 & $A$ & NS \\
\hline
\end{tabular}

\begin{tabular}{|c|c|c|c|c|c|c|c|c|}
\hline Periods & Month & Decades & Means $\left({ }^{\circ} \mathrm{C}\right)$ & Diff. $\left({ }^{\circ} \mathrm{C}\right)$ & F-ratio & P-value & H. G. & S.Level \\
\hline $1951-1980$ & July & 19 & 22.06 & & & & A & \\
\hline $1981-2010$ & July & 19 & 22.28 & 0.22 & 0.39 & 0.5372 & A & NS \\
\hline 1951-1980 & July & 20 & 21.66 & & & & A & \\
\hline $1981-2010$ & July & 20 & 22.32 & 0.66 & 5.38 & 0.0239 & B & $\mathrm{s}$ \\
\hline 1951-1980 & July & 21 & 21.6 & & & & A & \\
\hline 1981-2010 & July & 21 & 21.93 & 0.33 & 2.55 & $\begin{array}{r}0.1155 \\
\end{array}$ & A & NS \\
\hline 1951-1980 & August & 22 & 21.6 & & & & A & \\
\hline $1981-2010$ & August & 22 & 21.71 & 0.11 & 0.23 & 0.6323 & A & NS \\
\hline 1951-1980 & August & 23 & 21.47 & & & & A & \\
\hline $1981-2010$ & August & 23 & 21.8 & 0.33 & 2.39 & $\begin{array}{r}0.1273 \\
\end{array}$ & A & NS \\
\hline $1951-1980$ & August & 24 & 21.3 & & & & A & \\
\hline $1981-2010$ & August & 24 & 21.76 & 0.46 & 5.47 & $\begin{array}{r}0.0229 \\
-\end{array}$ & $B$ & $s$ \\
\hline 1951-1980 & September & 25 & 21.12 & & & & A & \\
\hline 1981-2010 & September & 25 & 21.42 & 0.3 & 0.30 & 0.1839 & A & NS \\
\hline 1951-1980 & September & _.26 & 21.27 & & & & A & \\
\hline $1981-2010$ & September & 26 & 21.71 & 0.44 & 5.01 & $\begin{array}{r}0.0290 \\
-\end{array}$ & $B$ & $s$ \\
\hline $1951-1980$ & September & 27 & 21.3 & & & & A & \\
\hline $1981-2010$ & September & 27 & 21.82 & 0.52 & 8.08 & 0.0062 & B & $\mathrm{s}$ \\
\hline $1951-1980$ & October & $\begin{array}{r}28 \\
-\end{array}$ & 21.09 & & & & A & \\
\hline $1981-2010$ & October & 28 & 21.64 & 0.55 & 7.52 & 0.0091 & $B$ & $\mathrm{~s}$ \\
\hline 1951-1980 & October & 29 & 20.87 & & & & A & \\
\hline $1981-2010$ & October & 29 & 21.48 & 0.61 & 6.02 & 0.0172 & $B$ & $\mathrm{~s}$ \\
\hline $1951-1980$ & October & $\begin{array}{r}30 \\
- \\
-\end{array}$ & -19.6 & & & & A & \\
\hline $1981-2010$ & October & 30 & 19.93 & 0.33 & 0.87 & 0.3537 & A & NS \\
\hline $1951-1980$ & November & 31 & 17.98 & & & & A & \\
\hline 1981-2010 & November & 31 & 18.11 & 0.13 & 0.08 & 0.7796 & A & NS \\
\hline 1951-1980 & November & 32 & 16.7 & & & & A & \\
\hline $1981-2010$ & November & 32 & 17.14 & 0.44 & 0.89 & 0.3486 & A & NS \\
\hline $1951-1980$ & November & 33 & 15.63 & & & & A & \\
\hline $1981-2010$ & November & 33 & 16.44 & 0.81 & 3.22 & 0.0778 & A & NS \\
\hline $1951-1980$ & December & 34 & 14.46 & & & & A & \\
\hline $1981-2010$ & December & 34 & 16.17 & 1.71 & 13.21 & 0.0006 & B & $\mathrm{s}$ \\
\hline 1951-1980 & December & 35 & 14.26 & & & & A & \\
\hline $1981-2010$ & December & 35 & 15.92 & 1.66 & 11.71 & 0.0011 & A & NS \\
\hline $1951-1980$ & December & $\begin{array}{r}36 \\
- \\
-\end{array}$ & 14.12 & & & & A & \\
\hline $1981-2010$ & December & 36 & 14.81 & 0.69 & 2.19 & 0.1445 & B & $\mathrm{s}$ \\
\hline \multicolumn{3}{|c|}{ Average Periods 1951-1980 } & 19.82 & & & & $A$ & \\
\hline \multicolumn{3}{|c|}{ Average Periods 1981-2010 } & 20.87 & 1.05 & 24.62 & 0.0000 & $\mathrm{~B}$ & $\mathrm{~s}$ \\
\hline
\end{tabular}


Table 8. Maximum temperature every ten days statistical analysis of the period 1951-1980 (30 years) and compared to the period 1981-2010 (30 years) at the meteorological station of Koutiala in Mali $\left(12.24{ }^{\circ} \mathrm{N}\right.$ latitude; $5.28^{\circ} \mathrm{O}$ longitude, altitude $334 \mathrm{~m}$

\begin{tabular}{|c|c|c|c|c|c|c|c|c|}
\hline Periods & Month & Decades & Means $\left({ }^{\circ} \mathrm{C}\right)$ & Diff. $\left({ }^{\circ} \mathrm{C}\right)$ & F-ratio & P-value & H.G. & S.Leve \\
\hline $1951-1980$ & January & 1 & 32.68 & \multirow[b]{2}{*}{-1.39} & \multirow[b]{2}{*}{9.54} & \multirow{2}{*}{0.0031} & A & \multirow[b]{2}{*}{$s$} \\
\hline $1981-2010$ & January & 1 & 31.29 & & & & $B$ & \\
\hline 1951-1980 & January & 2 & 32.8 & \multirow[b]{2}{*}{-0.87} & \multirow[b]{2}{*}{2.45} & \multirow{2}{*}{0.1229} & A & \multirow[b]{2}{*}{ NS } \\
\hline $1981-2010$ & January & 2 & 31.93 & & & & $A$ & \\
\hline $1951-1980$ & January & 3 & 33.89 & \multirow[b]{2}{*}{-0.83} & \multirow[b]{2}{*}{2.41} & \multirow[b]{2}{*}{0.1257} & A & \multirow[b]{2}{*}{ NS } \\
\hline $1981-2010$ & January & 3 & 33.06 & & & & A & \\
\hline $1951-1980$ & February & 4 & 34.74 & \multirow[b]{2}{*}{-0.31} & \multirow[b]{2}{*}{0.38} & \multirow{2}{*}{0.5418} & A & \multirow[b]{2}{*}{ NS } \\
\hline $1981-2010$ & February & 4 & 34.43 & & & & $A$ & \\
\hline $1951-1980$ & February & 5 & 36.3 & \multirow[b]{2}{*}{-0.78} & \multirow[b]{2}{*}{4.50} & \multirow[b]{2}{*}{0.0381} & A & \multirow[b]{2}{*}{$\mathrm{s}$} \\
\hline $1981-2010$ & February & 5 & 35.52 & & & & $B$ & \\
\hline 1951-1980 & February & 6 & 36.94 & \multirow[b]{2}{*}{-0.1} & \multirow[b]{2}{*}{0.08} & \multirow[b]{2}{*}{0.7815} & A & \multirow[b]{2}{*}{ NS } \\
\hline $1981-2010$ & February & 6 & 36.84 & & & & $A$ & \\
\hline $1951-1980$ & March & 7 & 37.28 & \multirow[b]{2}{*}{0.1} & \multirow[b]{2}{*}{0.00} & & A & \\
\hline $1981-2010$ & March & 7 & 37.38 & & & 1.0000 & $A$ & NS \\
\hline $1951-1980$ & March & 8 & 38.15 & & & & A & \\
\hline $1981-2010$ & March & 8 & 37.58 & -0.57 & 2.61 & 0.1117 & A & NS \\
\hline $1951-1980$ & March & 9 & 38.23 & & & & A & \\
\hline $1981-2010$ & March & 9 & 38.72 & 0.49 & 2.46 & 0.1225 & 5 & NS \\
\hline $1951-1980$ & April & 10 & 38.31 & & & & A & \\
\hline $1981-2010$ & April & 10 & 39.16 & 0.85 & 6.82 & 0.0114 & $4 \mathrm{~B}$ & $\mathrm{~s}$ \\
\hline $1951-1980$ & April & 11 & 38.39 & & & & A & \\
\hline $1981-2010$ & April & 11 & 38.96 & 0.57 & 3.10 & 0.0836 & $6 \mathrm{~A}$ & NS \\
\hline $1951-1980$ & April & 12 & 37.91 & & & & A & \\
\hline $1981-2010$ & April & 12 & 38.38 & 0.47 & 1.93 & 0.1704 & 4 & NS \\
\hline $1951-1980$ & May & 13 & 37.33 & & & & A & \\
\hline $1981-2010$ & May & 13 & 38.3 & 0.97 & 9.81 & 0.0027 & $7 \mathrm{~B}$ & $s$ \\
\hline 1951-1980 & May & 14 & 37.11 & & & & A & \\
\hline $1981-2010$ & May & 14 & 37.69 & 0.58 & 4.69 & 0.0305 & $5 \quad B$ & $\mathrm{~s}$ \\
\hline $1951-1980$ & May & 15 & 35.68 & & & & A & \\
\hline $1981-2010$ & May & 15 & 36.46 & 0.78 & 4.00 & 0.0501 & A & NS \\
\hline $1951-1980$ & June & 16 & 35.06 & & & & A & \\
\hline $1981-2010$ & June & 16 & 35.16 & 0.1 & 0.05 & 0.8241 & A & NS \\
\hline $1951-1980$ & June & 17 & 33.83 & & & & A & \\
\hline $1981-2010$ & June & 17 & 34.28 & 0.45 & 1.90 & 0.1733 & A & NS \\
\hline 1951-1980 & June & 18 & 33.02 & & & & A & \\
\hline $1981-2010$ & June & 18 & 32.85 & -0.17 & 0.47 & 0.4969 & 9 & NS \\
\hline
\end{tabular}

\begin{tabular}{|c|c|c|c|c|c|c|c|c|}
\hline Periods & Month & Decades & Means $\left({ }^{\circ} \mathrm{C}\right)$ & Diff. $\left({ }^{\circ} \mathrm{C}\right)$ & F-ratio & P-value & H. G. & S.Level \\
\hline $1951-1980$ & July & 19 & $92 \quad 32$ & \multirow[b]{2}{*}{0.01} & \multirow[b]{2}{*}{0.00} & \multirow[b]{2}{*}{0.9696} & A & \multirow[b]{2}{*}{ NS } \\
\hline $1981-2010$ & July & 19 & 32.01 & & & & $A$ & \\
\hline $1951-1980$ & July & 20 & 31 & \multirow[b]{2}{*}{0.35} & \multirow[b]{2}{*}{1.85} & \multirow{2}{*}{0.1796} & A & \multirow[b]{2}{*}{ NS } \\
\hline $1981-2010$ & July & 20 & 31.35 & & & & A & \\
\hline $1951-1980$ & July & 21 & 30.3 & \multirow[b]{2}{*}{0.19} & \multirow[b]{2}{*}{0.79} & \multirow{2}{*}{0.3788} & A & \multirow[b]{2}{*}{ NS } \\
\hline $1981-2010$ & July & 21 & 30.49 & & & & A & \\
\hline $1951-1980$ & August & 22 & 30.15 & \multirow[b]{2}{*}{0.11} & \multirow[b]{2}{*}{0.22} & \multirow[b]{2}{*}{0.6417} & A & \multirow[b]{2}{*}{ NS } \\
\hline $1981-2010$ & August & 22 & 30.26 & & & & A & \\
\hline 1951-1980 & August & 23 & 29.72 & \multirow[b]{2}{*}{0.45} & \multirow[b]{2}{*}{3.49} & \multirow[b]{2}{*}{0.0666} & A & \multirow[b]{2}{*}{ NS } \\
\hline $1981-2010$ & August & 23 & 30.17 & & & & A & \\
\hline $1951-1980$ & August & 24 & 29.91 & \multirow[b]{2}{*}{0.38} & \multirow[b]{2}{*}{2.20} & \multirow[b]{2}{*}{0.1432} & A & \multirow[b]{2}{*}{ NS } \\
\hline $1981-2010$ & August & 24 & 30.29 & & & & $A$ & \\
\hline $1951-1980$ & September & 25 & 30.38 & \multirow[b]{2}{*}{-0.01} & & & A & \\
\hline $1981-2010$ & September & 25 & 30.37 & & 0.01 & 0.9424 & A & NS \\
\hline $1951-1980$ & September & 26 & 31.06 & & & & A & \\
\hline $1981-2010$ & September & 26 & 31.44 & 0.38 & 2.57 & 0.1144 & $A$ & NS \\
\hline $1951-1980$ & September & 27 & 31.99 & & & & A & \\
\hline $1981-2010$ & September & 27 & 32.51 & 0.52 & 4.40 & 0.0403 & B & $\mathrm{s}$ \\
\hline 1951-1980 & October & 28 & 33.17 & & & & A & \\
\hline $1981-2010$ & October & 28 & 33.64 & 0.47 & 2.46 & 0.1221 & A & NS \\
\hline $1951-1980$ & October & 29 & 34.06 & & & & A & \\
\hline $1981-2010$ & October & 29 & 34.47 & 0.41 & 1.82 & 0.1823 & A & NS \\
\hline $1951-1980$ & October & 30 & 34.41 & & & & A & \\
\hline $1981-2010$ & October & 30 & 35.35 & 0.94 & 10.45 & 0.0020 & B & $\mathrm{s}$ \\
\hline $1951-1980$ & November & 31 & 34.91 & & & & A & \\
\hline $1981-2010$ & November & 31 & 35.58 & 0.67 & 7.31 & 0.0090 & B & $s$ \\
\hline $1951-1980$ & November & 32 & 34.97 & & & & A & \\
\hline $1981-2010$ & November & 32 & 35.3 & 0.33 & 2.06 & 0.1563 & A & NS \\
\hline $1951-1980$ & November & 33 & 34.07 & & & & A & \\
\hline $1981-2010$ & November & 33 & 34.94 & 0.87 & 6.40 & 0.0142 & B & $s$ \\
\hline $1951-1980$ & December & 34 & 33.34 & & & & A & \\
\hline $1981-2010$ & December & 34 & 33.62 & 0.28 & 0.59 & 0.4446 & A & NS \\
\hline $1951-1980$ & December & 35 & 32.51 & & & & A & \\
\hline $1981-2010$ & December & 35 & 32.95 & 0.44 & 0.87 & 0.3536 & A & NS \\
\hline 1951-1980 & December & 36 & 32.18 & & & & A & \\
\hline $1981-2010$ & December & 36 & 32.47 & 0.29 & 0.39 & 0.5300 & $A$ & NS \\
\hline Ave & ge Periods 19 & -1980 & 34.11 & & & & A & \\
\hline Ave & ge Periods 19 & -2010 & 34.3 & 0.19 & 3.20 & 0.0788 & $A$ & NS \\
\hline
\end{tabular}


Table 9. Minimum temperature every ten days statistical analysis of the period 1951-1980 (30 years) and compared to the period 1981-2010 (30 years) at the meteorological station of Koutiala in Mali. $\left(12.24{ }^{\circ} \mathrm{N}\right.$ latitude, $5.28^{\circ} \mathrm{O}$ longitude, altitude $334 \mathrm{~m}$ )

\begin{tabular}{|c|c|c|c|c|c|c|c|c|}
\hline Periods & Month & Decades & Means $\left({ }^{\circ} \mathrm{C}\right)$ & Diff. $\left({ }^{\circ} \mathrm{C}\right)$ & F-ratio & P-value & H.G. & S.Level \\
\hline $1951-1980$ & January & 1 & 12.75 & & & & A & \\
\hline $1981-2010$ & January & 1 & 15.47 & 2.72 & 19.37 & 0.0000 & B & $s$ \\
\hline $1951-1980$ & January & 2 & 12.93 & & & & A & \\
\hline $1981-2010$ & January & 2 & 16.28 & 3.35 & 39.16 & 0.0000 & B & $\mathrm{s}$ \\
\hline $1951-1980$ & January & 3 & 13.21 & & & & A & \\
\hline $1981-2010$ & January & 3 & 16.7 & 3.49 & 36.50 & 0.0000 & B & $s$ \\
\hline $1951-1980$ & February & 4 & 14.64 & & & & A & \\
\hline $1981-2010$ & February & 4 & 17.8 & 3.16 & 28.96 & 0.0000 & $B$ & $\mathrm{~s}$ \\
\hline $1951-1980$ & February & 5 & 15.9 & & & & A & \\
\hline $1981-2010$ & February & 5 & 19.31 & 3.41 & 22.72 & 0.0000 & B & $s$ \\
\hline $1951-1980$ & February & 6 & 17.06 & & & & A & \\
\hline $1981-2010$ & February & 6 & 20.43 & 3.37 & 35.23 & 0.0000 & $B$ & $\mathrm{~s}$ \\
\hline $1951-1980$ & March & 7 & 17.91 & & & & A & \\
\hline $1981-2010$ & March & 7 & 22.19 & 4.28 & 47.57 & 0.0000 & B & $\mathrm{s}$ \\
\hline $1951-1980$ & March & 8 & 19.95 & & & & A & \\
\hline $1981-2010$ & March & 8 & 23.4 & 3.45 & 30.48 & 0.0000 & B & $s$ \\
\hline $1951-1980$ & March & 9 & 21.74 & & & & A & \\
\hline $1981-2010$ & March & 9 & 24.34 & 2.6 & 19.14 & 0.0001 & B & $\mathrm{s}$ \\
\hline $1951-1980$ & April & 10 & 22.77 & & & & A & \\
\hline $1981-2010$ & April & 10 & 25.74 & 2.97 & 53.61 & 0.0000 & B & $\mathrm{s}$ \\
\hline $1951-1980$ & April & 11 & 23.79 & & & & A & \\
\hline $1981-2010$ & April & 11 & 26.54 & 2.75 & 53.66 & 0.0000 & $B$ & $\mathrm{~s}$ \\
\hline $1951-1980$ & April & 12 & 25.09 & & & & A & \\
\hline $1981-2010$ & April & 12 & 26.63 & 1.54 & 27.91 & 0.0000 & B & $\mathrm{s}$ \\
\hline $1951-1980$ & May & 13 & 24.78 & & & & A & \\
\hline $1981-2010$ & May & 13 & 26.6 & 1.82 & 47.87 & 0.0000 & $B$ & $\mathrm{~s}$ \\
\hline $1951-1980$ & May & 14 & 25.14 & & & & A & \\
\hline $1981-2010$ & May & 14 & 26.09 & 0.95 & 17.54 & 0.0001 & B & $\mathrm{s}$ \\
\hline 1951-1980 & May & 15 & 24.13 & & & & A & \\
\hline $1981-2010$ & May & 15 & 25.44 & 1.31 & 36.98 & 0.0000 & $B$ & $\mathrm{~s}$ \\
\hline $1951-1980$ & June & 16 & 23.74 & & & & A & \\
\hline $1981-2010$ & June & 16 & 24.47 & 0.73 & 7.07 & 0.0101 & $B$ & $\mathrm{~s}$ \\
\hline 1951-1980 & June & 17 & 23.06 & & & & A & \\
\hline $1981-2010$ & June & 17 & 23.88 & 0.82 & 13.73 & 0.0005 & $B$ & $\mathrm{~s}$ \\
\hline $1951-1980$ & June & 18 & 22.51 & & & & A & \\
\hline $1981-2010$ & June & 18 & 23.28 & 0.77 & 18.50 & 0.0001 & $B$ & $\mathrm{~s}$ \\
\hline
\end{tabular}

\begin{tabular}{|c|c|c|c|c|c|c|c|c|}
\hline Periods & Month & Decades & Means $\left({ }^{\circ} \mathrm{C}\right)$ & Diff. $\left({ }^{\circ} \mathrm{C}\right)$ & F-ratio & P-value & H. G. & S.Level \\
\hline 1951-1980 & July & 19 & 22.17 & & & & A & \\
\hline $1981-2010$ & July & 19 & 22.74 & 0.57 & 12.88 & 0.0007 & $B$ & $\mathrm{~s}$ \\
\hline 1951-1980 & July & 20 & 21.81 & & & & A & \\
\hline $1981-2010$ & July & 20 & 22.51 & 0.7 & 24.15 & 0.0000 & $B$ & $\mathrm{~s}$ \\
\hline 1951-1980 & July & 21 & 21.52 & & & & A & \\
\hline $1981-2010$ & July & 21 & 22.27 & 0.75 & 39.24 & 0.0000 & $B$ & $\mathrm{~s}$ \\
\hline 1951-1980 & August & 22 & 21.71 & & & & A & \\
\hline $1981-2010$ & August & 22 & 22.1 & 0.39 & 10.38 & 0.0021 & $B$ & $\mathrm{~s}$ \\
\hline 1951-1980 & August & 23 & 21.55 & & & & A & \\
\hline $1981-2010$ & August & 23 & 22.12 & 0.57 & 24.22 & 0.0000 & $B$ & $\mathrm{~s}$ \\
\hline 1951-1980 & August & 24 & 21.44 & & & & A & \\
\hline $1981-2010$ & August & 24 & 22.12 & 0.68 & 39.20 & 0.0000 & $B$ & $s$ \\
\hline \begin{tabular}{|l|}
$1951-1980$ \\
\end{tabular} & September & 25 & 21.27 & & & & A & \\
\hline $1981-2010$ & September & 25 & 21.95 & 0.68 & 25.76 & 0.0000 & $B$ & $\mathrm{~s}$ \\
\hline 1951-1980 & September & 26 & 21.3 & & & & A & \\
\hline $1981-2010$ & September & 26 & 22.13 & 0.83 & 42.41 & 0.0000 & $B$ & $s$ \\
\hline 1951-1980 & September & 27 & 21.64 & & & & A & \\
\hline $1981-2010$ & September & 27 & 22.58 & 0.94 & 45.21 & 0.0000 & $B$ & $\mathrm{~s}$ \\
\hline 1951-1980 & October & 28 & 21.52 & & & & A & \\
\hline $1981-2010$ & October & 28 & 22.69 & 1.17 & 65.45 & 0.0000 & $B$ & $s$ \\
\hline 1951-1980 & October & 29 & 21.49 & & & & A & \\
\hline $1981-2010$ & October & 29 & 22.93 & 1.44 & 52.06 & 0.0000 & $B$ & $\mathrm{~s}$ \\
\hline $1951-1980$ & October & 30 & 20.67 & & & & A & \\
\hline $1981-2010$ & October & 30 & 22.39 & 1.72 & 45.81 & 0.0000 & $B$ & $s$ \\
\hline 1951-1980 & November & 31 & 18.89 & & & & $A$ & \\
\hline $1981-2010$ & November & 31 & 20.44 & 1.55 & 14.63 & 0.0003 & $B$ & $\mathrm{~s}$ \\
\hline $1951-1980$ & November & 32 & 17.1 & & & & A & \\
\hline $1981-2010$ & November & 32 & 19.44 & 2.34 & 21.57 & 0.0000 & $B$ & $s$ \\
\hline 1951-1980 & November & 33 & 15.11 & & & & A & \\
\hline $1981-2010$ & November & 33 & 17.92 & 2.81 & 32.79 & 0.0000 & $B$ & $s$ \\
\hline \begin{tabular}{|l}
$1951-1980$ \\
\end{tabular} & December & 34 & 14.03 & & & & $A$ & \\
\hline $1981-2010$ & December & 34 & 17.24 & 3.21 & 40.34 & 0.0000 & $B$ & $s$ \\
\hline 1951-1980 & December & 35 & 13.44 & & & & A & \\
\hline $1981-2010$ & December & 35 & 16.62 & 3.18 & 40.20 & 0.0000 & $B$ & $s$ \\
\hline $1951-1980$ & December & 36 & 13.1 & & & & A & \\
\hline $1981-2010$ & December & 36 & 15.56 & 2.46 & 22.66 & 0.0000 & B & $\mathrm{s}$ \\
\hline \multicolumn{3}{|c|}{ Average Periods 1951-1980 } & 19.75 & & & & A & \\
\hline \multicolumn{3}{|c|}{ Average Periods 1981-2010 } & 21.68 & 1.93 & 110.62 & 0.0000 & $B$ & $\mathrm{~s}$ \\
\hline
\end{tabular}


Table 10. Maximum temperature every ten days statistical analysis of the period 1951-1980 (30 years) and compared to the period 1981-2010 (30 years) at the meteorological station of Sikasso in Mali $\left(11.21^{\circ} \mathrm{N}\right.$ latitude, $5.41^{\circ} \mathrm{O}$ longitude, altitude $\left.271 \mathrm{~m}\right)$

\begin{tabular}{|c|c|c|c|c|c|c|c|c|}
\hline Periods & Month & Decades & Means $\left({ }^{\circ} \mathrm{C}\right)$ & Diff. $\left({ }^{\circ} \mathrm{C}\right)$ & F-ratio & P-value & H. G. & S.Level \\
\hline $1951-1980$ & January & 1 & 33.52 & & & & A & \\
\hline $1981-2010$ & January & 1 & 31.87 & -1.65 & 17.18 & 0.0001 & B & s \\
\hline 1951-1980 & January & 2 & 33.64 & & & & A & \\
\hline $1981-2010$ & January & 2 & 32.56 & -1.08 & 5.33 & 0.0246 & B & $\mathrm{s}$ \\
\hline $1951-1980$ & January & 3 & 34.63 & & & & A & \\
\hline $1981-2010$ & January & 3 & 33.65 & -0.98 & 4.50 & 0.0381 & $B$ & $\mathrm{~s}$ \\
\hline $1951-1980$ & February & 4 & 35.3 & & & & A & \\
\hline $1981-2010$ & February & 4 & 34.78 & -0.52 & 1.42 & 0.2385 & A & NS \\
\hline $1951-1980$ & February & 5 & 36.58 & & & & A & \\
\hline $1981-2010$ & February & 5 & 35.64 & -0.94 & 9.09 & 0.0038 & $B$ & $\mathrm{~s}$ \\
\hline 1951-1980 & February & 6 & 37.14 & & & & A & \\
\hline $1981-2010$ & February & 6 & 36.95 & -0.19 & 0.46 & 0.5010 & $A$ & NS \\
\hline 1951-1980 & March & 7 & 37.24 & & & & A & \\
\hline $1981-2010$ & March & 7 & 37.02 & -0.22 & 0.54 & 0.4657 & A & NS \\
\hline $1951-1980$ & March & 8 & 37.73 & & & & A & \\
\hline $1981-2010$ & March & 8 & 37.11 & -0.62 & 3.70 & 0.0594 & $A$ & NS \\
\hline $1951-1980$ & March & 9 & 37.61 & & & & A & \\
\hline 1981-2010 & March & 9 & 37.86 & 0.25 & 0.92 & 0.3427 & $\bar{A}$ & NS \\
\hline 1951-1980 & April & 10 & 37.62 & & & & A & \\
\hline $1981-2010$ & April & 10 & 38 & 0.38 & 1.30 & 0.2583 & $\mathrm{~A}$ & NS \\
\hline $1951-1980$ & April & 11 & 37.38 & & & & A & \\
\hline $1981-2010$ & April & 11 & 37.25 & -0.13 & 0.22 & 0.6400 & A & NS \\
\hline $1951-1980$ & April & 12 & 36.73 & & & & A & \\
\hline $1981-2010$ & April & 12 & 36.59 & -0.14 & 0.16 & 0.6888 & $8 \mathrm{~A}$ & NS \\
\hline $1951-1980$ & May & 13 & 36.28 & & & & A & \\
\hline $1981-2010$ & May & 13 & 36.42 & 0.14 & 0.23 & 0.6345 & 5 & NS \\
\hline 1951-1980 & May & 14 & 35.82 & & & & A & \\
\hline $1981-2010$ & May & 14 & 35.64 & -0.18 & 0.48 & 0.4904 & 4 & NS \\
\hline $1951-1980$ & May & 15 & 34.72 & & & & A & \\
\hline $1981-2010$ & May & 15 & 34.8 & 0.08 & 0.04 & 0.8367 & $7 \mathrm{~A}$ & NS \\
\hline 1951-1980 & June & 16 & 33.95 & & & & A & \\
\hline $1981-2010$ & June & 16 & 33.44 & -0.51 & 2.34 & 0.1312 & $2 \mathrm{~A}$ & NS \\
\hline $1951-1980$ & June & 17 & 33.08 & & & & A & \\
\hline $1981-2010$ & June & 17 & 32.9 & -0.18 & 0.50 & 0.4811 & $1 \mathrm{~A}$ & NS \\
\hline 1951-1980 & June & 18 & 32.53 & & & & A & \\
\hline $1981-2010$ & June & 18 & 31.76 & -0.77 & 11.09 & 0.0015 & B & $\mathrm{s}$ \\
\hline
\end{tabular}

\begin{tabular}{|c|c|c|c|c|c|c|c|c|}
\hline Periods & Month & Decades & Means $\left({ }^{\circ} \mathrm{C}\right)$ & Diff. $\left({ }^{\circ} \mathrm{C}\right)$ & F-ratio & P-value & H. G. & S.Level \\
\hline $1951-1980$ & July & 19 & 9 & \multirow[b]{2}{*}{-0.58} & \multirow[b]{2}{*}{5.67} & \multirow[b]{2}{*}{0.0206} & A & \multirow[b]{2}{*}{$\mathrm{s}$} \\
\hline $1981-2010$ & July & 19 & 31.04 & & & & B & \\
\hline $1951-1980$ & July & 20 & 30.91 & \multirow[b]{2}{*}{-0.36} & \multirow[b]{2}{*}{2.40} & \multirow[b]{2}{*}{0.1270} & A & \multirow[b]{2}{*}{ NS } \\
\hline $1981-2010$ & July & 20 & 30.55 & & & & A & \\
\hline $1951-1980$ & July & 21 & 30.23 & \multirow[b]{2}{*}{-0.24} & \multirow[b]{2}{*}{1.08} & \multirow[b]{2}{*}{0.3019} & A & \multirow[b]{2}{*}{ NS } \\
\hline $1981-2010$ & July & 21 & 29.99 & & & & $A$ & \\
\hline 1951-1980 & August & 22 & 30.15 & \multirow[b]{2}{*}{-0.37} & \multirow[b]{2}{*}{2.49} & \multirow[b]{2}{*}{0.1197} & A & \multirow[b]{2}{*}{ NS } \\
\hline $1981-2010$ & August & 22 & 29.78 & & & & A & \\
\hline 1951-1980 & August & 23 & 29.72 & \multirow[b]{2}{*}{0.03} & \multirow[b]{2}{*}{0.03} & \multirow[b]{2}{*}{0.8714} & A & \multirow[b]{2}{*}{ NS } \\
\hline $1981-2010$ & August & 23 & 29.75 & & & & A & \\
\hline $1951-1980$ & August & 24 & 29.99 & \multirow[b]{2}{*}{-0.08} & \multirow[b]{2}{*}{0.12} & \multirow[b]{2}{*}{0.7315} & A & \multirow[b]{2}{*}{ NS } \\
\hline $1981-2010$ & August & 24 & 29.91 & & & & A & \\
\hline 1951-1980 & September & 25 & 30.27 & \multirow[b]{2}{*}{-0.03} & & & A & \\
\hline $1981-2010$ & September & 25 & 30.24 & & 0.02 & 0.8810 & A & NS \\
\hline $1951-1980$ & September & 26 & 31.13 & & & & A & \\
\hline $1981-2010$ & September & 26 & 30.95 & -0.18 & 0.61 & 0.4395 & A & NS \\
\hline $1951-1980$ & September & 27 & 31.94 & & & & A & \\
\hline $1981-2010$ & September & 27 & 31.76 & -0.18 & 0.57 & 0.4541 & A & NS \\
\hline $1951-1980$ & October & 28 & 32.76 & & & & A & \\
\hline $1981-2010$ & October & 28 & 32.54 & -0.22 & 0.67 & 0.4151 & A & NS \\
\hline $1951-1980$ & October & 29 & 33.62 & & & & A & \\
\hline $1981-2010$ & October & 29 & 33.2 & -0.42 & 2.64 & 0.1401 & A & NS \\
\hline $1951-1980$ & October & 30 & 33.84 & & & & A & \\
\hline $1981-2010$ & October & 30 & 34.04 & 0.2 & 0.46 & 0.5160 & A & NS \\
\hline 1951-1980 & November & 31 & 34.39 & & & & A & \\
\hline $1981-2010$ & November & 31 & 34.39 & 0 & 0.00 & 0.9880 & A & NS \\
\hline $1951-1980$ & November & 32 & 34.6 & & & & A & \\
\hline $1981-2010$ & November & 32 & 34.58 & -0.02 & 0.01 & 0.9166 & A & NS \\
\hline 1951-1980 & November & 33 & 34.05 & & & & A & \\
\hline $1981-2010$ & November & 33 & 34.38 & 0.33 & 1.46 & 0.2312 & $A$ & NS \\
\hline 1951-1980 & December & 34 & 33.66 & & & & A & \\
\hline $1981-2010$ & December & 34 & 33.41 & -0.25 & 0.82 & 0.3687 & A & NS \\
\hline $1951-1980$ & December & 35 & 33.05 & & & & A & \\
\hline $1981-2010$ & December & 35 & 33.04 & -0.01 & 0.00 & 0.9714 & A & NS \\
\hline 1951-1980 & December & 36 & 33.14 & & & & A & \\
\hline $1981-2010$ & December & 36 & 32.68 & -0.46 & 1.46 & 0.2324 & $A$ & NS \\
\hline & ge Periods 19 & -1980 & 33.62 & & & & A & \\
\hline Av & age Periods 19 & -2010 & 33.9 & 0.28 & 6.66 & 0.0124 & B & $\mathrm{s}$ \\
\hline
\end{tabular}


Table 11. Minimum temperature every ten days statistical analysis of the period 1951-1980 (30 years) and compared to the period 1981-2010 (30 years) at the meteorological station of Sikasso in Mali $\left(11.21^{\circ} \mathrm{N}\right.$ latitude, $5.41^{\circ} \mathrm{O}$ longitude, altitude $\left.271 \mathrm{~m}\right)$

\begin{tabular}{|c|c|c|c|c|c|c|c|c|}
\hline Periods & Month & Decades & Means $\left({ }^{\circ} \mathrm{C}\right)$ & Diff. $\left({ }^{\circ} \mathrm{C}\right)$ & F-ratio & P-value & H. G. & S.Level \\
\hline $1951-1980$ & January & 1 & 14.05 & & & & A & \\
\hline $1981-2010$ & January & 1 & 15.86 & 1.81 & 14.87 & 0.0003 & B & $\mathrm{s}$ \\
\hline $1951-1980$ & January & 2 & 14.36 & & & & A & \\
\hline $1981-2010$ & January & 2 & 16.89 & 2.53 & 25.51 & 0.0000 & B & $\mathrm{s}$ \\
\hline $1951-1980$ & January & 3 & 14.84 & & & & A & \\
\hline $1981-2010$ & January & 3 & 17.56 & 2.72 & 31.45 & 0.0000 & B & $\mathrm{s}$ \\
\hline 1951-1980 & February & 4 & 16.31 & & & & A & \\
\hline $1981-2010$ & February & 4 & 18.32 & 2.01 & 26.03 & 0.0000 & $B$ & $\mathrm{~s}$ \\
\hline $1951-1980$ & February & 5 & 17.72 & & & & A & \\
\hline $1981-2010$ & February & 5 & 20.31 & 2.59 & 17.14 & 0.0001 & B & $s$ \\
\hline 1951-1980 & February & 6 & 19.17 & & & & A & \\
\hline $1981-2010$ & February & 6 & 21.46 & 2.29 & 20.31 & 0.0000 & $B$ & $\mathrm{~s}$ \\
\hline $1951-1980$ & March & 7 & 19.6 & & & & A & \\
\hline $1981-2010$ & March & 7 & 22.73 & 3.13 & 27.24 & 0.0000 & B & $\mathrm{s}$ \\
\hline $1951-1980$ & March & 8 & 21.67 & & & & A & \\
\hline $1981-2010$ & March & 8 & 23.72 & 2.05 & 23.47 & 0.0000 & B & $\mathrm{s}$ \\
\hline 1951-1980 & March & 9 & 22.87 & & & & A & \\
\hline $1981-2010$ & March & 9 & 24.53 & 1.66 & 13.91 & 0.0004 & B & $\mathrm{s}$ \\
\hline $1951-1980$ & April & 10 & 23.58 & & & & A & \\
\hline $1981-2010$ & April & 10 & 25.45 & 1.87 & 56.05 & 0.0000 & B & $\mathrm{s}$ \\
\hline 1951-1980 & April & 11 & 24.01 & & & & A & \\
\hline $1981-2010$ & April & 11 & 25.72 & 1.71 & 26.28 & 0.0000 & $B$ & $\mathrm{~s}$ \\
\hline $1951-1980$ & April & 12 & 24.59 & & & & A & \\
\hline $1981-2010$ & April & 12 & 25.17 & 0.58 & 4.85 & 0.0317 & B & $\mathrm{s}$ \\
\hline $1951-1980$ & May & 13 & 24.17 & & & & A & \\
\hline 1981-2010 & May & 13 & 25.01 & 0.84 & 13.72 & 0.0005 & B & $\mathrm{s}$ \\
\hline $1951-1980$ & May & 14 & 24.08 & & & & A & \\
\hline $1981-2010$ & May & 14 & 24.62 & 0.54 & 5.92 & 0.0181 & $B$ & $\mathrm{~s}$ \\
\hline 1951-1980 & May & 15 & 23.07 & & & & A & \\
\hline 1981-2010 & May & 15 & 24.24 & 1.17 & 25.85 & 0.0000 & $\mathrm{~B}$ & $\mathrm{~s}$ \\
\hline 1951-1980 & June & 16 & 22.88 & & & & A & \\
\hline 1981-2010 & June & 16 & 23.25 & 0.37 & 3.84 & 0.0550 & A & NS \\
\hline 1951-1980 & June & 17 & 22.11 & & & & A & \\
\hline $1981-2010$ & June & 17 & 22.88 & 0.77 & 14.67 & 0.0003 & $B$ & $\mathrm{~s}$ \\
\hline 1951-1980 & June & 18 & 21.77 & & & & A & \\
\hline $1981-2010$ & June & 18 & 22.41 & 0.64 & 11.96 & 0.0010 & $B$ & $\mathrm{~s}$ \\
\hline
\end{tabular}

\begin{tabular}{|c|c|c|c|c|c|c|c|c|}
\hline Periods & Month & Decades & Means $\left({ }^{\circ} \mathrm{C}\right)$ & Diff. $\left({ }^{\circ} \mathrm{C}\right)$ & F-ratio & P-value & H. G. & S.Level \\
\hline 1951-1980 & July & 19 & 21.56 & & & & A & \\
\hline 1981-2010 & July & 19 & 22.23 & 0.67 & 11.18 & 0.0015 & $B$ & $s$ \\
\hline $1951-1980$ & July & 20 & 21.36 & & & & A & \\
\hline $1981-2010$ & July & 20 & 21.96 & 0.6 & 10.02 & 0.0025 & B & $s$ \\
\hline $1951-1980$ & July & 21 & 21.2 & & & & A & \\
\hline $1981-2010$ & July & 21 & 21.88 & 0.68 & 25.80 & 0.0000 & B & $\mathrm{s}$ \\
\hline $1951-1980$ & August & 22 & 21.38 & & & & A & \\
\hline $1981-2010$ & August & 22 & 21.67 & 0.29 & 4.22 & 0.0445 & $B$ & $s$ \\
\hline $1951-1980$ & August & 23 & 21.3 & & & & A & \\
\hline 1981-2010 & August & 23 & 21.64 & 0.34 & 13.10 & 0.0006 & $B$ & $s$ \\
\hline 1951-1980 & August & 24 & 21.21 & & & & A & \\
\hline $1981-2010$ & August & 24 & 21.61 & 0.4 & 16.78 & 0.0001 & $B$ & $\mathrm{~s}$ \\
\hline $1951-1980$ & September & 25 & 21.06 & & & & A & \\
\hline $1981-2010$ & September & 25 & 21.37 & 0.31 & 5.20 & 0.0263 & B & $s$ \\
\hline $1951-1980$ & September & 26 & 21.13 & & & & A & \\
\hline 1981-2010 & September & 26 & 21.5 & 0.37 & 10.07 & 0.0024 & $B$ & $s$ \\
\hline $1951-1980$ & September & 27 & 21.18 & & & & A & \\
\hline $1981-2010$ & September & 27 & 21.68 & 0.5 & 22.29 & 0.0000 & B & $\mathrm{s}$ \\
\hline $1951-1980$ & October & 28 & 21.38 & & & & A & \\
\hline $1981-2010$ & October & 28 & 21.91 & 0.53 & 17.90 & 0.0001 & $B$ & $\mathrm{~s}$ \\
\hline 1951-1980 & October & 29 & 21.48 & & & & A & \\
\hline $1981-2010$ & October & 29 & 22.11 & 0.63 & 20.73 & 0.0000 & $B$ & $\mathrm{~s}$ \\
\hline $1951-1980$ & October & 30 & 21.09 & & & & A & \\
\hline $1981-2010$ & October & 30 & 21.79 & 0.7 & 12.13 & 0.0009 & B & $\mathrm{s}$ \\
\hline 1951-1980 & November & 31 & 19.74 & & & & A & \\
\hline 1981-2010 & November & 31 & 20.38 & 0.64 & 1.27 & 0.2637 & A & NS \\
\hline 1951-1980 & November & 32 & 18.56 & & & & A & \\
\hline $1981-2010$ & November & 32 & 19.35 & 0.79 & 2.91 & 0.0935 & A & NS \\
\hline $1951-1980$ & November & 33 & 16.2 & & & & A & \\
\hline $1981-2010$ & November & 33 & 18.15 & 1.95 & 16.89 & 0.0001 & $B$ & $\mathrm{~s}$ \\
\hline 1951-1980 & December & 34 & 14.97 & & & & A & \\
\hline $1981-2010$ & December & 34 & 17.32 & 2.35 & 21.46 & 0.0000 & $B$ & $\mathrm{~s}$ \\
\hline $1951-1980$ & December & 35 & 14.4 & & & & A & \\
\hline $1981-2010$ & December & 35 & 16.73 & 2.33 & 23.81 & 0.0000 & $B$ & $\mathrm{~s}$ \\
\hline 1951-1980 & December & 36 & 14.1 & & & & A & \\
\hline $1981-2010$ & December & 36 & 16.08 & 1.98 & 20.37 & 0.0000 & $B$ & $\mathrm{~s}$ \\
\hline \multicolumn{3}{|c|}{ Average Periods 1951-1980 } & 20.12 & & & & A & \\
\hline \multicolumn{3}{|c|}{ Average Periods $1981-2010$} & 21.37 & 1.25 & 79.26 & 0.0000 & B & $\mathrm{s}$ \\
\hline
\end{tabular}


Table 12. Maximum temperature every ten days statistical analysis of the period 1951-1980 (30 years) and compared to the period 1981-2010 (30 years) at the meteorological station of Bougouni in Mali $\left(11.25^{\circ} \mathrm{N}\right.$ latitude, $7.30^{\circ} \mathrm{O}$ longitude, altitude $350 \mathrm{~m}$ )

\begin{tabular}{|c|c|c|c|c|c|c|c|c|}
\hline Periode & Month & Decades & Means $\left({ }^{\circ} \mathrm{C}\right)$ & Diff. $\left({ }^{\circ} \mathrm{C}\right)$ & F-ratio & P-value & H.G. & S.Level \\
\hline $1951-1980$ & January & 1 & 33.45 & & & & A & \\
\hline $1981-2010$ & January & 1 & 32.85 & -0.6 & 2.09 & 0.1540 & A & NS \\
\hline $1951-1980$ & January & 2 & 33.5 & & & & A & \\
\hline $1981-2010$ & January & 2 & 33.34 & -0.16 & 0.12 & 0.7321 & A & NS \\
\hline $1951-1980$ & January & 3 & 34.47 & & & & A & \\
\hline $1981-2010$ & January & 3 & 34.43 & -0.04 & 0.00 & 0.9447 & A & NS \\
\hline $1951-1980$ & February & 4 & 35.1 & & & & A & \\
\hline $1981-2010$ & February & 4 & 35.75 & 0.65 & 2.15 & 0.1480 & A & NS \\
\hline $1951-1980$ & February & 5 & 36.27 & & & & A & \\
\hline $1981-2010$ & February & 5 & 36.58 & 0.31 & 1.16 & 0.2857 & A & NS \\
\hline $1951-1980$ & February & 6 & 36.87 & & & & A & \\
\hline $1981-2010$ & February & 6 & 37.84 & 0.97 & 10.83 & 0.0017 & $B$ & s \\
\hline $1951-1980$ & March & 7 & 37.02 & & & & A & \\
\hline $1981-2010$ & March & 7 & 38 & 0.98 & 11.88 & 0.0011 & $B$ & $s$ \\
\hline $1951-1980$ & March & 8 & 37.44 & & & & A & \\
\hline $1981-2010$ & March & 8 & 38.24 & 0.8 & 5.50 & 0.0225 & $B$ & $s$ \\
\hline $1951-1980$ & March & 9 & 37.55 & & & & A & \\
\hline $1981-2010$ & March & 9 & 38.75 & 1.2 & 18.90 & 0.0001 & $B$ & $s$ \\
\hline $1951-1980$ & April & 10 & 37.53 & & & & A & \\
\hline $1981-2010$ & April & 10 & 39.06 & 1.53 & 23.88 & 0.0000 & $B$ & $s$ \\
\hline $1951-1980$ & April & 11 & 37.33 & & & & A & \\
\hline $1981-2010$ & April & 11 & 38.62 & 1.29 & 18.26 & 0.0001 & $B$ & $s$ \\
\hline $1951-1980$ & April & 12 & 36.45 & & & & A & \\
\hline $1981-2010$ & April & 12 & 37.51 & 1.06 & 7.84 & 0.0069 & $B$ & $s$ \\
\hline $1951-1980$ & May & 13 & 36.04 & & & & A & \\
\hline $1981-2010$ & May & 13 & 37.6 & 1.56 & 16.92 & 0.0001 & $B$ & $s$ \\
\hline $1951-1980$ & May & 14 & 35.34 & & & & A & \\
\hline $1981-2010$ & May & 14 & 36.73 & 1.39 & 12.25 & 0.0009 & $B$ & $\mathrm{~s}$ \\
\hline $1951-1980$ & May & 15 & 34.26 & & & & A & \\
\hline $1981-2010$ & May & 15 & 35.52 & 1.26 & 9.85 & 0.0027 & $B$ & $s$ \\
\hline $1951-1980$ & June & 16 & 33.51 & & & & A & \\
\hline $1981-2010$ & June & 16 & 34.24 & 0.73 & 3.53 & 0.0654 & A & NS \\
\hline $1951-1980$ & June & 17 & 32.39 & & & & A & \\
\hline $1981-2010$ & June & 17 & 33.6 & 1.21 & 16.93 & 0.0001 & $B$ & $s$ \\
\hline $1951-1980$ & June & 18 & 31.9 & & & & A & \\
\hline $1981-2010$ & June & 18 & 32.6 & 0.7 & 7.20 & 0.0095 & $B$ & $\mathrm{~s}$ \\
\hline
\end{tabular}

\begin{tabular}{|c|c|c|c|c|c|c|c|c|}
\hline Periode & Month & Decades & Means $\left({ }^{\circ} \mathrm{C}\right)$ & Diff. $\left({ }^{\circ} \mathrm{C}\right)$ & F-ratio & P-value & H. G. & S.Level \\
\hline $1951-1980$ & July & $\begin{array}{r}19 \\
\end{array}$ & $\begin{array}{r}1.06 \\
\end{array}$ & & & & $A$ & \\
\hline $1981-2010$ & July & 19 & 31.65 & 0.59 & 4.82 & 0.0321 & B & $\mathrm{s}$ \\
\hline $1951-1980$ & July & 20 & 30.25 & & & & A & \\
\hline $1981-2010$ & July & 20 & 31.04 & 0.79 & 10.20 & 0.0023 & B & $\mathrm{s}$ \\
\hline $1951-1980$ & July & 21 & 29.63 & & & & $A$ & \\
\hline $1981-2010$ & July & 21 & 30.63 & 1 & 13.93 & 0.0004 & B & $\mathrm{s}$ \\
\hline 1951-1980 & August & 22 & 29.58 & & & & A & \\
\hline $1981-2010$ & August & 22 & 30.36 & 0.78 & 10.73 & 0.0018 & B & $\mathrm{s}$ \\
\hline $1951-1980$ & August & 23 & 29.22 & & & & A & \\
\hline $1981-2010$ & August & 23 & 30.34 & 1.12 & 26.84 & 0.0000 & B & $\mathrm{s}$ \\
\hline 1951-1980 & August & 24 & 29.45 & & & & A & \\
\hline $1981-2010$ & August & 24 & 30.48 & 1.03 & 25.18 & 0.0000 & B & $\mathrm{s}$ \\
\hline $1951-1980$ & September & 25 & 29.92 & & & & A & \\
\hline $1981-2010$ & September & 25 & 30.59 & 0.67 & 10.92 & 0.0016 & B & s \\
\hline 1951-1980 & September & 26 & 30.49 & & & & A & \\
\hline $1981-2010$ & September & 26 & 31.44 & 0.95 & 22.25 & 0.0000 & B & $\mathrm{s}$ \\
\hline 1951-1980 & September & 27 & 31.18 & & & & A & \\
\hline $1981-2010$ & September & 27 & 32.07 & 0.89 & 17.72 & 0.0001 & B & $\mathrm{s}$ \\
\hline 1951-1980 & October & 28 & 32.16 & & & & A & \\
\hline $1981-2010$ & October & 28 & 32.71 & 0.55 & 3.51 & 0.0662 & $A$ & NS \\
\hline $1951-1980$ & Octoober & 29 & 32.8 & & & & A & \\
\hline $1981-2010$ & October & 29 & 33.44 & 0.64 & 5.12 & 0.0274 & B & $\mathrm{s}$ \\
\hline $1951-1980$ & October & 30 & 33.13 & & & & A & \\
\hline $1981-2010$ & October & 30 & 34.15 & 1.02 & 15.23 & 0.0003 & B & $\mathrm{s}$ \\
\hline $1951-1980$ & November & 31 & 33.78 & & & & A & \\
\hline $1981-2010$ & November & 31 & 34.93 & 1.15 & 21.65 & 0.0000 & B & $\mathrm{s}$ \\
\hline 1951-1980 & November & 32 & 34.14 & & & & A & \\
\hline $1981-2010$ & November & 32 & 35.17 & 1.03 & 18.04 & 0.0001 & B & $\mathrm{s}$ \\
\hline $1951-1980$ & November & 33 & 33.87 & & & & A & \\
\hline $1981-2010$ & November & 33 & 35.16 & 1.29 & 20.79 & 0.0000 & B & $\mathrm{s}$ \\
\hline 1951-1980 & December & 34 & 33.56 & & & & A & \\
\hline $1981-2010$ & December & 34 & 34.27 & 0.71 & 6.13 & 0.0162 & B & $\mathrm{s}$ \\
\hline 1951-1980 & December & 35 & 32.88 & & & & A & \\
\hline $1981-2010$ & December & 35 & 33.87 & 0.99 & 5.81 & 0.0191 & $B$ & $\mathrm{~s}$ \\
\hline 1951-1980 & December & 36 & 32.84 & & & & A & \\
\hline $1981-2010$ & December & 36 & 33.56 & 0.72 & 2.77 & 0.1015 & $B$ & $\mathrm{~s}$ \\
\hline \multicolumn{3}{|c|}{ Average Periods 1951-1980 } & 33.51 & & & & A & \\
\hline \multicolumn{3}{|c|}{ Average Periods 1981-2010 } & 34.36 & 0.85 & 36.59 & 0.0000 & B & $\mathrm{s}$ \\
\hline
\end{tabular}


Table 13. Minimum temperature every ten days statistical analysis of the period 1951-1980 (30 years) and compared to the period 1981-2010 (30 years) at the meteorological station of Bougouni in Mali $\left(11.25^{\circ} \mathrm{N}\right.$ latitude, $7.30^{\circ} \mathrm{O}$ longitude, altitude $350 \mathrm{~m}$ )

\begin{tabular}{|c|c|c|c|c|c|c|c|c|}
\hline Periode & Month & Decades & Means $\left({ }^{\circ} \mathrm{C}\right)$ & Diff. $\left({ }^{\circ} \mathrm{C}\right)$ & F-ratio & P-value & H. G. & S.Level \\
\hline $1951-1980$ & January & 1 & 15.67 & & & & A & \\
\hline $1981-2010$ & January & 1 & 15.91 & 0.24 & 0.33 & 0.5702 & A & NS \\
\hline $1951-1980$ & January & 2 & 15.98 & & & & $A$ & \\
\hline $1981-2010$ & January & 2 & 16.79 & 0.81 & 3.75 & 0.0578 & A & NS \\
\hline $1951-1980$ & January & 3 & 16.68 & & & & A & \\
\hline $1981-2010$ & January & 3 & 17.37 & 0.69 & 2.26 & 0.1383 & A & NS \\
\hline $1951-1980$ & February & 4 & 17.86 & & & & A & \\
\hline $1981-2010$ & February & 4 & 18.71 & 0.85 & 4.08 & 0.0479 & $B$ & $\mathrm{~s}$ \\
\hline $1951-1980$ & February & 5 & 18.92 & & & & A & \\
\hline $1981-2010$ & February & 5 & 19.95 & 1.03 & 3.92 & 0.0525 & A & NS \\
\hline $1951-1980$ & February & 6 & 20.2 & & & & A & \\
\hline $1981-2010$ & February & 6 & 21.03 & 0.83 & 3.26 & 0.0761 & A & NS \\
\hline $1951-1980$ & March & 7 & 20.6 & & & & A & \\
\hline $1981-2010$ & March & 7 & 22.4 & 1.8 & 14.75 & 0.0003 & B & $\mathrm{s}$ \\
\hline $1951-1980$ & March & 8 & 22.16 & & & & A & \\
\hline $1981-2010$ & March & 8 & 23.25 & 1.09 & 7.16 & 0.0097 & B & $\mathrm{s}$ \\
\hline $1951-1980$ & March & 9 & 23.36 & & & & A & \\
\hline $1981-2010$ & March & 9 & 23.98 & 0.62 & 2.85 & 0.0970 & A & NS \\
\hline $1951-1980$ & April & 10 & 24.01 & & & & A & \\
\hline $1981-2010$ & April & 10 & 25.04 & 1.03 & 13.68 & 0.0005 & B & $\mathrm{s}$ \\
\hline $1951-1980$ & April & 11 & 24.25 & & & & A & \\
\hline $1981-2010$ & April & 11 & 25.9 & 1.65 & 43.34 & 0.0000 & $B$ & $\mathrm{~s}$ \\
\hline $1951-1980$ & April & 12 & 24.59 & & & & A & \\
\hline $1981-2010$ & April & 12 & 25.28 & 0.69 & 9.51 & 0.0031 & B & $\mathrm{s}$ \\
\hline $1951-1980$ & May & 13 & 24.22 & & & & A & \\
\hline $1981-2010$ & May & 13 & 25.33 & 1.11 & 21.53 & 0.0000 & $B$ & $s$ \\
\hline $1951-1980$ & May & 14 & 24.04 & & & & A & \\
\hline $1981-2010$ & May & 14 & 24.74 & 0.7 & 9.66 & 0.0029 & B & s \\
\hline $1951-1980$ & May & 15 & 23.22 & & & & A & \\
\hline $1981-2010$ & May & 15 & 24.23 & 1.01 & 21.91 & 0.0000 & $B$ & $\mathrm{~s}$ \\
\hline $1951-1980$ & June & 16 & 22.79 & & & & A & \\
\hline $1981-2010$ & June & 16 & 23.41 & 0.62 & 8.87 & 0.0042 & B & $\mathrm{s}$ \\
\hline $1951-1980$ & June & 17 & 22.15 & & & & A & \\
\hline $1981-2010$ & June & 17 & 22.96 & 0.81 & 23.08 & 0.0000 & B & $\mathrm{s}$ \\
\hline $1951-1980$ & June & 18 & 21.82 & & & & A & \\
\hline $1981-2010$ & June & 18 & 22.6 & 0.78 & 28.91 & 0.0000 & B & $\mathrm{s}$ \\
\hline
\end{tabular}

\begin{tabular}{|c|c|c|c|c|c|c|c|c|}
\hline Periode & Month & Decades & Means $\left({ }^{\circ} \mathrm{C}\right)$ & Diff. $\left({ }^{\circ} \mathrm{C}\right)$ & F-ratio & P-value & H. G. & S.Level \\
\hline $1951-1980$ & July & 19 & 21.53 & & & & A & \\
\hline $1981-2010$ & July & 19 & 22.15 & 0.62 & 17.06 & 0.0001 & B & $s$ \\
\hline 1951-1980 & July & 20 & 21.37 & & & & A & \\
\hline $1981-2010$ & July & 20 & 21.95 & 0.58 & 18.41 & 0.0001 & B & $\mathrm{s}$ \\
\hline 1951-1980 & July & 21 & 21.28 & & & & A & \\
\hline 1981-2010 & July & 21 & 21.97 & 0.69 & 33.53 & 0.0000 & B & $s$ \\
\hline $1951-1980$ & August & $\begin{array}{r}22 \\
-- \\
\end{array}$ & $\begin{array}{r}21.36 \\
\end{array}$ & & & & A & \\
\hline $1981-2010$ & August & 22 & 21.88 & 0.52 & 16.44 & 0.0002 & B & $\mathrm{s}$ \\
\hline 1951-1980 & August & 23 & 21.26 & & & & A & \\
\hline $1981-2010$ & August & 23 & 21.87 & 0.61 & 29.05 & 0.0000 & B & $s$ \\
\hline $1951-1980$ & August & 24 & 21.09 & & & & A & \\
\hline $1981-2010$ & August & 24 & 21.7 & 0.61 & 20.93 & 0.0000 & B & $s$ \\
\hline 1951-1980 & September & 25 & 21.09 & & & & A & \\
\hline 1981-2010 & September & 25 & 21.7 & 0.61 & 20.93 & 0.0000 & B & $\mathrm{s}$ \\
\hline 1951-1980 & September & _.26 & 21.11 & & & & A & \\
\hline $1981-2010$ & September & 26 & 21.64 & 0.53 & 18.84 & 0.0001 & $B$ & $\mathrm{~s}$ \\
\hline $1951-1980$ & September & 27 & 21.2 & & & & A & \\
\hline $1981-2010$ & September & 27 & 21.75 & 0.55 & 20.65 & 0.0000 & B & $\mathrm{s}$ \\
\hline $1951-1980$ & October & $\begin{array}{r}28 \\
-\end{array}$ & 21.22 & & & & A & \\
\hline $1981-2010$ & October & 28 & 21.83 & 0.61 & 18.64 & 0.0001 & B & $\mathrm{s}$ \\
\hline $1951-1980$ & October & 29 & 21.24 & & & & A & \\
\hline $1981-2010$ & October & 29 & 22.01 & 0.77 & 311.08 & 0.0000 & B & $\mathrm{s}$ \\
\hline $1951-1980$ & October & $\begin{array}{r}30 \\
- \\
-\end{array}$ & 20.92 & & & & A & \\
\hline $1981-2010$ & October & 30 & 21.55 & 0.63 & 15.55 & 0.0002 & B & $\mathrm{s}$ \\
\hline 1951-1980 & November & 31 & 19.81 & & & & A & \\
\hline $1981-2010$ & November & 31 & 19.83 & 0.02 & 0.00 & 0.9619 & A & NS \\
\hline $1951-1980$ & November & 32 & 18.55 & & & & A & \\
\hline $1981-2010$ & November & 32 & 18.58 & 0.03 & 0.01 & 0.9327 & A & NS \\
\hline $1951-1980$ & November & 33 & 16.82 & & & & A & \\
\hline $1981-2010$ & November & 33 & 17.31 & 0.49 & 1.51 & 0.2237 & A & NS \\
\hline $1951-1980$ & December & 34 & 15.77 & & & & A & \\
\hline $1981-2010$ & December & 34 & 16.23 & 0.46 & 0.99 & 0.3245 & A & NS \\
\hline 1951-1980 & December & 35 & 15.47 & & & & A & \\
\hline $1981-2010$ & December & 35 & 15.5 & 0.03 & 0.00 & 0.9718 & A & NS \\
\hline $1951-1980$ & December & $\begin{array}{r}36 \\
- \\
-\end{array}$ & 14.99 & & & & A & \\
\hline $1981-2010$ & December & 36 & 15.43 & 0.44 & 0.50 & 0.4817 & A & NS \\
\hline \multicolumn{3}{|c|}{ Average Periods 1951-1980 } & 20.53 & & & & A & \\
\hline \multicolumn{3}{|c|}{ Average Periods 1981-2010 } & 21.21 & 0.68 & 30.15 & 0.0000 & $\mathrm{~B}$ & $\mathrm{~s}$ \\
\hline
\end{tabular}

\section{Copyrights}

Copyright for this article is retained by the author(s), with first publication rights granted to the journal.

This is an open-access article distributed under the terms and conditions of the Creative Commons Attribution license (http://creativecommons.org/licenses/by/3.0/). 